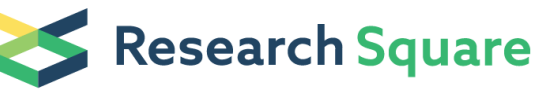 \\ Preprints are preliminary reports that have not undergone peer review. \\ They should not be considered conclusive, used to inform clinical practice, or referenced by the media as validated information.
}

\section{Novel Detection of Diesel Adulteration using Silver coated Surface Plasmon Resonance Sensor}

\section{Md. Asaduzzaman Jabin}

MBSTU: Mawlana Bhashani Science and Technology University

Md. Juwel Rana

MBSTU: Mawlana Bhashani Science and Technology University

Fahad Ahmed Al-Zahrani

Umm Al-Qura University

\section{Bikash Kumar Paul}

MBSTU: Mawlana Bhashani Science and Technology University

Kawsar Ahmed (D kawsar.ict@mbstu.ac.bd)

Mawlana Bhashani Science and Technology University https://orcid.org/0000-0002-4034-9819

Francis Minhthang Bui

University of Saskatchewan

\section{Research Article}

Keywords: Biophotonics, Diesel Adulteration Sensing, Optical Fiber Biosensor, Silver-Coated Biosensor, Surface Plasmon Resonance Sensor.

Posted Date: May 4th, 2021

DOl: https://doi.org/10.21203/rs.3.rs-440861/v1

License: (c) (i) This work is licensed under a Creative Commons Attribution 4.0 International License. Read Full License

Version of Record: A version of this preprint was published at Plasmonics on September 28th, 2021. See the published version at https://doi.org/10.1007/s11468-021-01540-4. 


\title{
Novel Detection of Diesel Adulteration using Silver coated Surface Plasmon Resonance Sensor
}

\author{
Md. Asaduzzaman Jabin ${ }^{1}$, Md. Juwel Rana ${ }^{1}$, Fahad Ahmed Al-Zahrani ${ }^{2}$, Bikash Kumar Paul ${ }^{1}$, \\ Kawsar Ahmed ${ }^{1, *}$, Francis Minhthang Bui ${ }^{3}$ \\ ${ }^{1}$ Group of Bio-photomati $\chi$, Department of Information and Communication Technology (ICT), \\ Mawlana Bhashani Science and Technology University (MBSTU), Tangail 1902, Bangladesh \\ ${ }^{2}$ Computer Engineering Department, Umm Al-Qura University, Mecca 24381, Saudi Arabia \\ ${ }^{3}$ Department of Electrical and Computer Engineering, University of Saskatchewan, 57 Campus \\ Drive, Saskatoon, SK S7N 5A9, Canada \\ *Corresponding Author: kawsar.ict@mbstu.ac.bd; k.ahmed.bd@ieee.org, kawsarit08050@gmail.com \\ *https://orcid.org/0000-0002-4034-9819
}

\begin{abstract}
In this study, we propose a silver-coated (SPR-surface plasmon resonance) based biosensor for the detection of diesel adulteration for the first time in the field of bio-sensing. Here, the numerical analysis is done by COMSOL Multiphysics V-5.1 and fully simulation software MATLAB-V16 on 30190 mesh elements, 2792 boundary elements, and 206593 degrees of freedom using finite element method (FEM) at $25^{\circ} \mathrm{C}(298 \mathrm{~K})$ for different concentration level. Moreover, the entire experiment on SPR-PCF is done for the major optical parameters for instanceBirefringence $\left(B_{i}\right)$, Coupling-length $\left(L_{c}\right)$, Power-fraction $\left(P_{f}\right)$, Confinement-loss $\left(\alpha_{c}\right)$, Amplitudesensitivity $\left(S_{a}\right)$, Wavelength-sensitivity $\left(S_{w}\right)$, Resolution $\left(R_{l}\right)$, Transmittance $\left(T_{x}\right)$, Transmittancevariance $\left(T_{v}\right)$, Relative-sensitivity $\left(S_{r}\right)$, Figure-of-merit $(F O M)$ and Resonance $\left(R^{2}\right)$ etc which are respectively to their corresponding maximum performance profiles of $2.5 \times 10^{-3}, 1300 \mu \mathrm{m}$, $99.93 \%, 1200-1600 \mathrm{~dB} / \mathrm{cm},-7950 R I U^{-1}, 49941.169 \mathrm{~nm} / \mathrm{RIU}, 6.319 \times 10^{-4} \mathrm{RIU},-180 \mathrm{~dB}$, $170 \mathrm{~dB} / \mathrm{RIU}, 97.25 \%, 980$, and 0.98312 at operating wavelength $0.5-2 \mu \mathrm{m}$. Therefore, it is noticed that the proposed sensor can be able to establish itself as one of the toughest candidates in fiber sensing. Also, diesel adulteration sensing including the entire working procedure using a SPR platform will be regarded as a novel procedure for diesel sensing in the history of photonics.
\end{abstract}

Keywords Biophotonics; Diesel Adulteration Sensing; Optical Fiber Biosensor; Silver-Coated Biosensor; Surface Plasmon Resonance Sensor.

\section{Introduction}

Nowadays, optical techniques for label-free detection of molecular interactions, binding, surface plasmon resonance (SPR) analysis has become very popular. In fact, surface plasmon has become a trending topic for its extraordinary performance profile and outfields like- cheapness, flexibility, durability and ultra-high sensitivity. Moreover, it acquires the leading position in sensing for example- disease diagnostics [1-2], cancer sensing [3], hemoglobin sensing [4], glucose sensing [5], chemical sensing [6], environmental monitoring [7], gas and liquid sensing [8] and water testing [9] etc. Moreover, the rapid improvement of optoelectronics for instance- SPR sensor [1, 3, 4], 
optical sensor [4-8] and THz bands sensor [10] are trending topics in photonics. As a result, SPR PCF is applied in bio-medical engineering with different analytical techniques for instance- molecular identification [11], nano-fluid [12], electro-chemical analysis [13] and immune-chemical fluid [14] etc. So, Industry level application of SPR-PCF can be possible and it is an ongoing process.

The ongoing development process of SPR based sensing started in the 1950s. In fact, in the same year, R. Richie et al. [15] introduced an optical-based SPR sensor for the very first time ever in the history of photonics. And, B. Liedberg et al. [16] proposed a SPR which has tremendous applications in remote-sensing because of its prism base. Nonetheless, RC. Jorgenson [17-18] showed a SPR-PCF with a gold coating nano-film with high sensing profiles that had extremely low peak resonance than any other optical sensors. Consequently, few upgradations in the structure are needed on-demand to get a better sensing profile. However, A. Rifat et al. [19] depicted a goldbased SPR PCF with a maximum sensing performance of near about $1000 \mathrm{~nm} / R I U$. Moreover, J.N. Dash et al. [17] proposed a SPR-PCF based sensor with the maximum sensing profile of almost $2200 \mathrm{~nm} / R I U$ and maximum amplitude-sensitivity of nearly $266 \mathrm{RIU}^{-1}$. And, B. Shuai et al. [20] explained a SPR-PCF based sensor for a wider refractive-index $(R I)$ profile of analytes. Later, X. Yang et al. [5] proposed a silver-coated biosensor for a chemical sensing performance of nearly $19009.17 \mathrm{~nm} / R I U$. Moreover, C. Liu et al. [21] exclaimed a gold-graphene composite film coating SPR PCF with better sensing performance. Besides, Rapid popularity has been attracted by PCF based SPR sensor due to its unique characteristics like outstanding sensing profile, low loss, high modal birefringence and moderate power confinement [22]. Optimum evanescent fields and desirable peak resonance are both easily derived from it [23]. It is kept in the note that PCF based SPR sensor provides high sensor resolution [23].

However, diesel has optical responsiveness in specific wavelengths due to its hydrocarbon chains. And, we are targeting this feature to introduce a novel SPR platform in diesel sensing platform. Therefore, diesel is regarded as the most commonly used fuel all over the world and the most valuable fuel product in the middle-east fuel export business. Nowadays, fuel adulteration is examined by the electrical sensors with major shortcomings like- costly, delay in sensing, electrical noise, magnetic resonance, and less re-usability, less accuracy, etc. To minimize those flaws, we have established an optical SPR platform, which has a tremendous profile with more accuracy, cost-effectiveness, re-usability, faster sensing, and noiseless features, etc.

In summary, the proposed SPR-PCF has better sensing profiles as well as better fabrication techniques. However, the refractive indices of fuel vary with the level of adulteration (due to its variation in the carbon chain) is depicted in Table. 1. In that case, our SPR PCF based sensor could be the right choice form ensuring the adulteration in the most effective, cheapest, implementable and reliable option for the very first time in the history of both chemical and bio-sensing.

\section{The Geometry and Internal structure of SPR PCF}

The cross-sectional view of the proposed diesel sensor is displayed in Fig. 1. Therefore, the corecladding boundary as well as PML is constructed with fused silica as a base material. Besides, it can be described with 3 layers likely- outer layer, inner layer and an intermediate layer. Moreover, the outer layer is described as a perfectly matched layer (PML) having the diameter of $d_{1}=5.80$ $\mu \mathrm{m}$ and the intermediate layer consists of analyte layer forms with the diameter of $d_{2}=5.235 \mu \mathrm{m}$. Therefore, the rest of the portion contains a cladding layer with a diameter of $d_{3}=4.60 \mu \mathrm{m}$ as well as a plasmonic metal layer of silver $(\mathrm{Ag})$ having a thickness of $t_{g}=35 \mathrm{~nm}$. Also, two rectangular air holes along with the same measurements of $x_{1}=3.80 \mu \mathrm{m}$, and $y_{1}=0.50 \mu \mathrm{m}$ and pitch-constant 
$P=2.0 \mu \mathrm{m}$ forms a cutting edge chord at the inner layer that helps to increase the area of analyte layer.
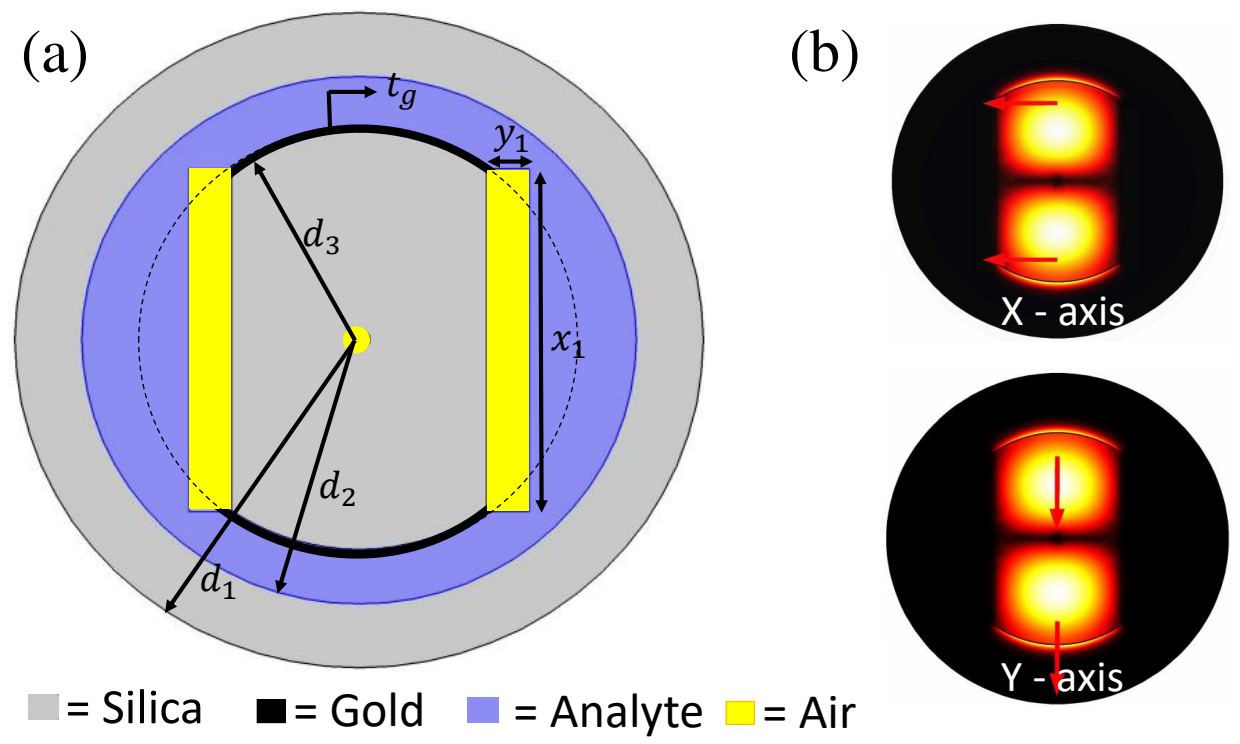

Fig. 1. (a) The internal diagram of SPR-PCF diesel adulteration measurement bio-sensor along with its geometrical parameters likely as $d_{1}=5.80 \mu \mathrm{m}, d_{2}=5.235 \mu \mathrm{m}, d_{3}=4.60 \mu \mathrm{m}, t_{g}=35 \mathrm{~nm}$, $x_{1}=3.80 \mu \mathrm{m}, y_{1}=0.50 \mu \mathrm{m}$ and pitch-constant $P=2.0 \mu \mathrm{m}$. And, $(b)$ The modes confinement of respectively for $x$ and $y$-polarization.

To sum up, the internal geometry contains a rectangular and circular structure that makes it implementable by using existing technology for instance- sol-gel, extrusion, and drilling methods that help to overcome all the issues (material selection, processing condition, alloy bonding technique, melting procedure, drilling, fabrication failure, and fiber dicing [1]-[23]) of real-life implementation.

\section{Numerical analysis}

The entire numerical analysis depends on polarization modes. Therefore, the proposed sensor depicts 2 polarization modes: (i) x-axis pole, and (ii) $y$-axis pole for spp modes, core modes, and transmission modes as well. Basically, the entire numerical analysis is done on user-defined mesh elements and starts with refractive indices analysis. The base material of the suggested sensor is fused silica and its refractive index is varied by wavelength $\lambda$ and some constants. So, Sellmeier's empirical equation is described as [25],

$$
n(\lambda)=\sqrt{\left(1+\frac{B_{1} \lambda^{2}}{\lambda^{2}-C_{1}}+\frac{B_{2} \lambda^{2}}{\lambda^{2}-C_{2}}+\frac{B_{3} \lambda^{2}}{\lambda^{2}-C_{3}}\right)}
$$

Here, the constants term used for fused silica are described as the Sellmeier's constants which are respectively for $0.69616,0.407942,0.897479,0.0046914826 \mu \mathrm{m}^{2}, 0.0135120631 \mu^{2}$ and 97.9340025 $\mu^{2}$ corresponding to $B_{1}, B_{2}, B_{3}, C_{1}, C_{2}$, and $C_{3}$.

However, both the carrier-sender and the receiver-sender interactions totally depend on modal 
birefringence profiles of the proposed PCF model that is calculated by the contrast of indices of polarization modes [26, 27],

$$
B_{i}=\Delta n_{x y}=\left|n_{x}^{i}-n_{y}^{i}\right|
$$

Here, $n_{x}$ and $n_{y}$ define the effective RI for the fundamental $\mathrm{x}$ and y-polarizations respectively.

In fact, the minimum fiber length at which the maximum amount of electromagnetic power of light interaction occurs through the fiber core is determined as the coupling-length and can be estimated by [27],

$$
L_{c}(\mu m)=\frac{\lambda}{2\left|n_{x}^{i}-n_{y}^{i}\right|}=\frac{\lambda}{B_{i}}
$$

Where, $B_{i}$ defines as the birefringence of the suggested model at the proposed operating wavelength.

However, the entire evanescent field's power-spectrum confines through the core of the structure. And, for calculating the mode confinement, the total output-power-spectrum (OPS) that passes through the core of silica-based fiber [28] can be examined by,

$$
P_{\text {out }}(w a t t)=\sin ^{2}\left(\frac{\left|n_{x}-n_{y}\right| \pi L}{\lambda}\right)=\sin ^{2}\left(\frac{\Delta n_{x y} \pi L}{\lambda}\right)
$$

Here, $L=$ the assumed length of the SPR-PCF. Nonetheless, the total efficiency of the fiber is examined by the power-fraction and [28] can be expressed by,

$$
P_{f}=\frac{P_{\text {in }}}{P_{\text {out }}}
$$

Here, output and input optical power is distinctly presented by $P_{\text {in }}$ and $P_{\text {out }} . P_{f}$ is the ration of input power over the output power. However, the entire mode-confinement creates a huge power loss that may be varied for mediums to mediums and [27] can be evaluated by,

$$
\alpha_{c}(d B / c m)=\operatorname{Im}\left[n_{e f f}\right] \times 8.686 \times \frac{2 \pi}{\lambda} \times 10^{4}
$$

Here, $\lambda$ defines as the operating wavelength for the proposed structure.

On the other hand, the total plasmonic incidents greatly rely on the transmittance profiles of the nominated PCF based SPR-PCF sensor. So, the transmittance performance [28] of the proposed sensor can be defined by,

$$
T_{x}(d B)=10 \times \log _{10}\left(\frac{P_{\text {out }}}{P_{\text {in }}}\right)
$$

Where, $P_{i n}$ defines as the maximum power fraction. Transmittance variance $T_{v}$ [28] can be calculated by,

$$
T_{v}(d B / R I U)=\frac{\max \left(t x_{b 1}-t x_{b 2}\right)}{\max \left(n_{b 1}-n_{b 2}\right)}
$$

Here, $\Delta n_{b}$ defines as the refractive-index (RI) difference between 2 transmission modes (i) xaxis polarization and (ii) y-axis polarization, and $t x_{(b 1, b 2)}$ defines as the maximum amplitude of transmittance $\left(T_{x}\right)$ profile for $b 1$ and $b 2$ respectively for the assumed analytes. Here, the absolute difference between two fundamental polarizations can be express by $\Delta n_{b}$ and $n x_{(b 1, b 2)}$ defines as 
the RI profiles at which the maximum amplitude of transmittance $T_{x}$ takes place respectively to $b 1$ and $b 2$ for the assumed analytes.

But, the overall problems of the amplitude-interrogation-method (AIM) can be minimized by amplitude sensitivity evaluation. The amplitude sensitivity of the proposed sensor can be evaluated by [27],

$$
S_{a}\left(R I U^{-1}\right)=-\frac{1}{\alpha_{c}} \times \frac{\Delta \alpha_{c}}{\Delta n}
$$

Where, $\alpha_{c}$ and $\Delta \alpha_{c}=$ the confinement losses profile and loss deviation for different diesel concentrations respectively, and $\Delta n$ defines as the refractive index (RI) contrast between the $x$ and $y$ polarization.

However, the wavelength-sensitivity can be determined through the steep peak of loss profile for an operating wavelength according to the variation of the refractive index (RI). And, the wavelength sensing $[27,28]$ profile for different diesel concentration can be defined as,

$$
S_{w}(n m / R I U)=\frac{\Delta \lambda_{p}}{\Delta n}
$$

Here, $\Delta \lambda p$ defines as the variety of wavelengths for different peaks and $\Delta n=$ the variation of refractive index (RI) of different diesel mixtures. On the other hand, any alteration the occurred in the RI of diesel can easily be noticeable by the amplitude-resolution variation of the proposed PCF. So, the entire resolution of the proposed PCF should be expressed by [27],

$$
R l(R I U)=\frac{\Delta n \times \Delta \lambda_{\text {min }}}{\Delta \lambda_{\text {peak }}}
$$

Here, $\Delta n$ defines as the RI difference. And, $\Delta \lambda_{\text {min }}$, and $\Delta \lambda_{\text {peak }}$ are respectively for the minimum wavelength contrast and peak wavelength-difference for a specific refractive index of the $\mathrm{x}$ and $\mathrm{y}$-axis polarization. Moreover, the relative-sensitivity is totally dependent on the effective refractive index profile (RI). And, the relative-sensitivity of the suggested PCF based SPR sensor can be measured by varying the index profile of core in it can be expressed by [29]

$$
R s(\%)=\frac{n_{\text {core }}}{n_{\text {eff }}} \times P_{\text {out }}
$$

Here, $n_{\text {core }}$ and $n_{\text {eff }}$ define the effective refractive index of core and effective refractive index of operating wavelength, and $P_{\text {out }}$ denotes as the power-spectrum of the output power.

Nonetheless, the actual measurement of the efficiency of the sensor is depicted by the FigureOf-Merit (FOM) and can be shown by [30],

$$
F O M=\frac{m\left(e V R I U^{-1}\right)}{F W H M P(e V)}
$$

Here, $m=$ the peak resonance slope per refractive index unit (RIU) and FWHMP defines as the half of maximum peak resonance full-width properties. These above-mentioned methods play a significant role to estimate sensor performances. Now based on these methodologies the results and discussion part are included below. 


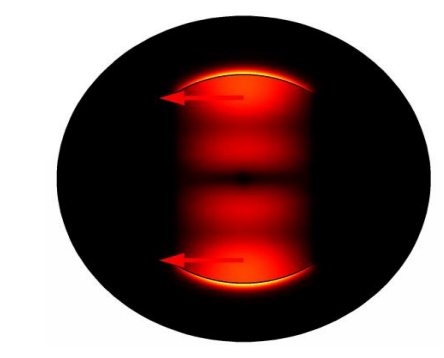

(a)

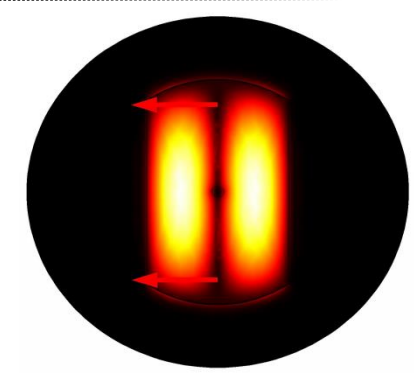

(c)

(b)

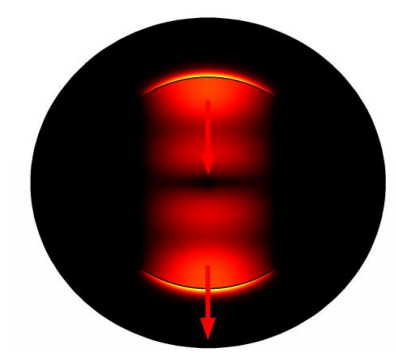

(d)

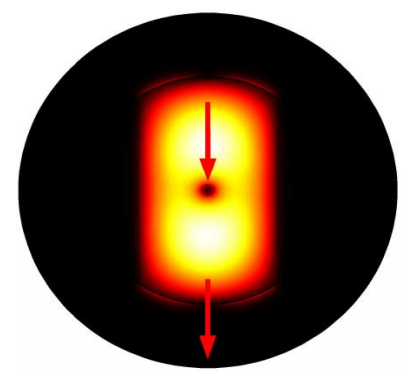

Fig. 2. (a-b) The surface plasmon (SPP) mode and (c-d) The mode-confinement for the core of the proposed structures for $x$-y axis polarization respectively.

Table 1 The group refractive index (RI) profiles for different adulteration levels of diesel with kerosene.

\begin{tabular}{|l|l|l|}
\hline $\begin{array}{l}\text { Concentration } \\
\text { of kerosene } \\
\text { in diesel (\% } \\
\text { v/v) }\end{array}$ & $\begin{array}{l}\text { Refractive } \\
\text { index of } \\
\text { mixtures }\end{array}$ & Reference \\
\hline 10 & 1.4660 & \\
20 & 1.4640 & \\
30 & 1.4620 & {$[31]$} \\
40 & 1.4600 & \\
50 & 1.4580 & \\
\hline
\end{tabular}

\section{Results and Discussion}

Fig. 3 exhibits the effective refractive index (RI) variation vs. the operating wavelength compared to the density variation of oil mixtures. In fact, it depicts a straight downward shifting curve ranges from $0.50 \mu \mathrm{m}$ to $2.00 \mu \mathrm{m}$ wavelength with a mild bend at the curve portion of the specific wavelength. For all density levels of diesel mixtures, the maximum effective refractive index (RI) varies between 1.42 and 1.47. To sum up, the x-polarization delineates the larger RI profiles compared to y-polarization and lower concentration levels $(10 \%)$ of diesel maintains a better refractive index (RI) profiles than any other concentration levels.

Fig. 4 exhibits the birefringence variation alteration respectively to the operating wavelength for different density levels of diesel mixtures. Moreover, birefringence is absolutely proportional to the shifting of wavelength. Particularly, the lower density level allows the minimal birefringence profile and vice versa. And, at the same optimum point of $1.50 \mu \mathrm{m}$ wavelength, the achieved birefringence profile is nearly $1.39 \times 10^{-3}, 1.365 \times 10^{-3}, 1.275 \times 10^{-3}$ and $1.245 \times 10^{-3}$ respectively to $10 \%, 20 \%, 30 \%, 40 \%$ and $50 \%$ density level. It is evident that the whole birefringence profile holds slower upward when $\lambda$ is shifting from $0.50 \mu \mathrm{m}$ to $0.80 \mu \mathrm{m}$, sharp upward shifting between 


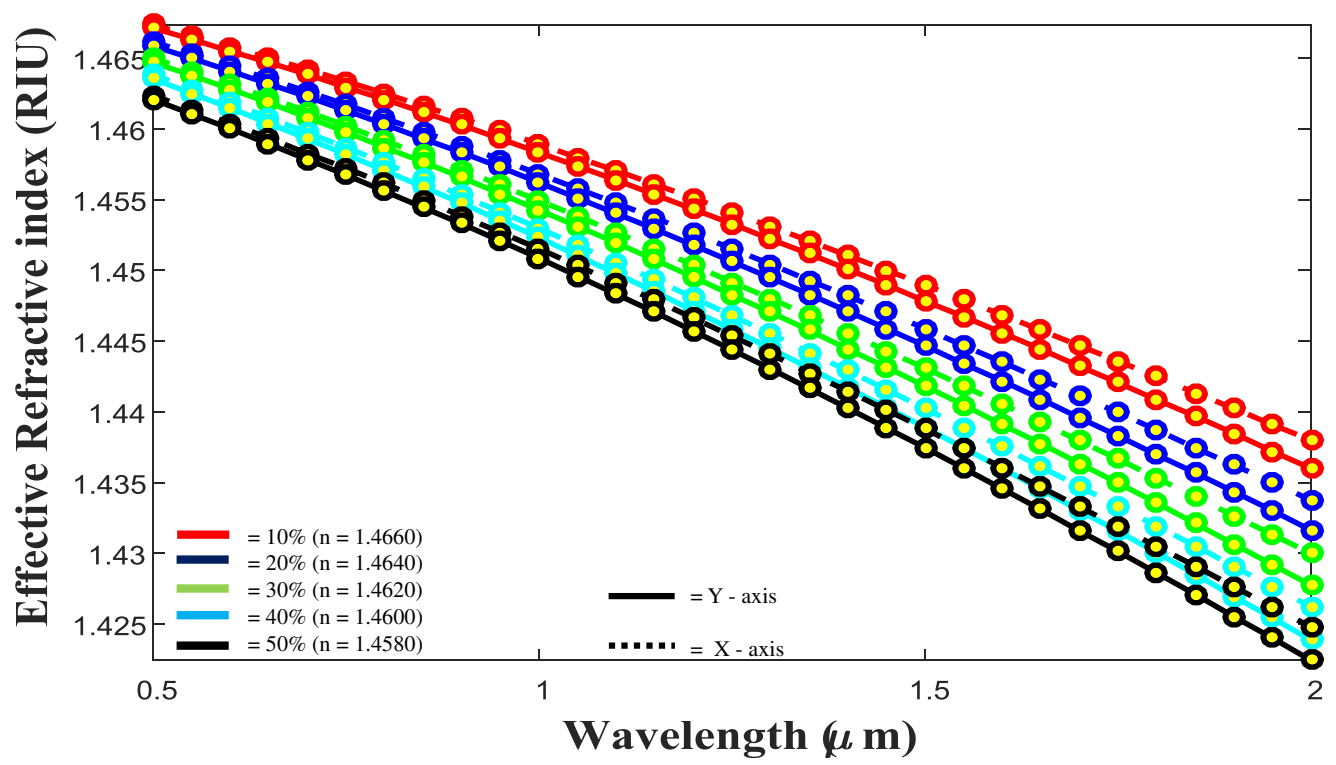

Fig. 3. The effective refractive index (RI) profile vs. operating wavelength for the proposed sensor for $x$-y axis polarization for different concentrations of adulteration level.

Table 2 The maximum possible sensing performance and the adulteration measurements of the PCF sensor.

\begin{tabular}{|l|l|l|l|l|l|}
\hline $\begin{array}{l}(\% \\
\mathrm{v} / \mathrm{v})\end{array}$ & $\begin{array}{l}R_{x} \\
(\%)\end{array}$ & $\begin{array}{l}S_{w} \\
(\mathrm{~nm} / \mathrm{RIU})\end{array}$ & $\begin{array}{l}S_{a} \\
\left(R I U^{-1}\right)\end{array}$ & $\mathrm{RI}(\mathrm{RIU})$ & $\begin{array}{l}P_{f} \\
(\%)\end{array}$ \\
\hline 10 & 97.25 & 49941.17 & -3550 & $6.32 \times 10^{-4}$ & 99.93 \\
20 & 97.19 & 40176.47 & -4510 & $5.97 \times 10^{-4}$ & 92.10 \\
30 & 97.15 & 35358.82 & -5902 & $5.22 \times 10^{-4}$ & 99.92 \\
40 & 97.08 & 31294.11 & -7810 & $3.77 \times 10^{-4}$ & 99.93 \\
50 & 97.06 & 29611.76 & -7950 & $2.31 \times 10^{-4}$ & 87.91 \\
\hline
\end{tabular}

$1.6 \mu \mathrm{m}$ and $2 \mu \mathrm{m}$ wavelength and rest displays moderate upward shifting. To sum up, a higher concentration level maintains a higher birefringence profile and so on.

Fig. 5 delineates the alteration of the coupling-length respectively to the controlling wavelength for the introduced micro-structure SPR-PCF. Also, the entire coupling length profiles of the proposed PCF sensor delineates a smooth downward trending curve-line with a slight bending manner. Besides, it varies for the maximum values of the range between $1300 \mu \mathrm{m}$ and 1000 $\mu \mathrm{m}$ that smoothly moves downward with a concave bend to the minimum values of ranges from $1000 \mu \mathrm{m}$ to $700 \mu \mathrm{m}$. Furthermore, it is examined that $10 \%$ adulteration of diesel displays the minimum coupling-length and that ranges between $950 \mu \mathrm{m}$ and $480 \mu \mathrm{m}$. Besides, $50 \%$ shows the lowest coupling-length varies from $1300 \mu \mathrm{m}$ to $700 \mu \mathrm{m}$. In fact, the coupling-length profiles of the proposed PCF depict a smoothly downward manner curve respectively to the linear manner of wavelength.

Fig. 6 shows power-spectrum variations vs. wavelength variation which follows the rhythm of a sinusoidal curve. From this figure, it is noticed that the power-spectrum of $50 \%$ density level of adulteration is lagged most and $10 \%$ density level of adulteration is forward mostly by a few phase-angles than the rest of power spectrum. The power spectrum amplitude varies inside the range of $(0,1)$. It is noticed that the power of the $50 \%$ adulteration level provides a dense spectrum 
Table 3 The maximum possible major performance profile of the adulteration measurement sensor.

\begin{tabular}{|l|l|l|l|l|}
\hline $\begin{array}{l}(\% \\
\mathrm{v} / \mathrm{v})\end{array}$ & $\begin{array}{l}T_{x} \\
(\mathrm{~dB})\end{array}$ & $\begin{array}{l}T_{v} \\
(\mathrm{~dB} / \mathrm{RIU})\end{array}$ & FOM & $\begin{array}{l}\text { Maximum } \\
\text { Detection } \\
\text { Limit }\end{array}$ \\
\hline 10 & -180 & 170 & 940 & 0.002 \\
20 & -140 & 135 & 945 & 0.002 \\
30 & -120 & 165 & 950 & 0.002 \\
40 & -115 & 165 & 970 & 0.002 \\
50 & -105 & 110 & 980 & 0.002 \\
\hline
\end{tabular}

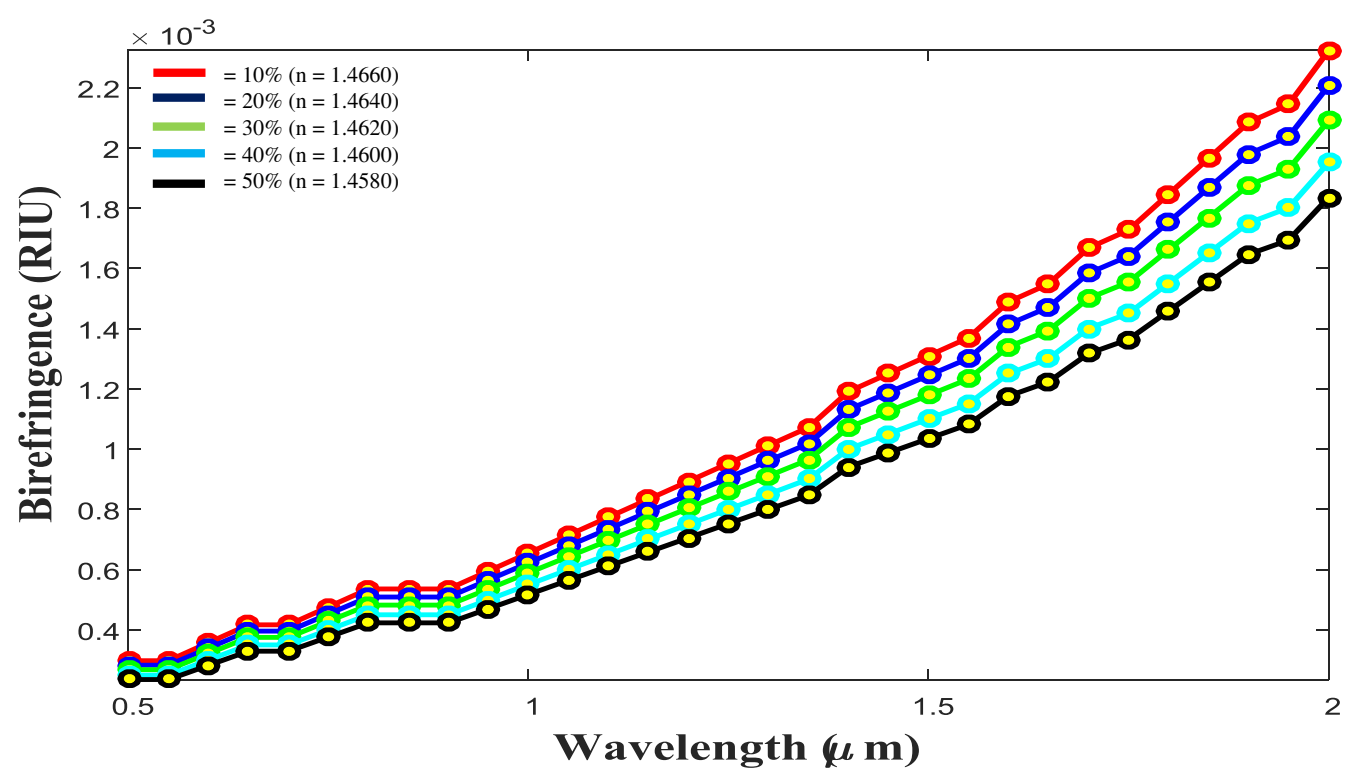

Fig. 4. The modal-birefringence performance of the proposed SPR-PCF oil adulteration sensor for different concentrations of adulteration level.

than the others.

Fig. 7 describes the power fraction as a function of operating wavelength with the range of wavelength from $0.5 \mu \mathrm{m}$ to $2.0 \mu \mathrm{m}$. Besides, it displays a smooth upward bending curve in a concave manner. However, the power fraction can be varied for the minimum values ranges from $87.91 \%$ to $35 \%$ and the maximum values varies between $99.93 \%$ and $57.41 \%$. Nonetheless, it is seen that the power fraction of $40 \%$ adulteration provides a better profile than others.

Fig. 8 depicts the chromatic confinement loss vs. wavelength respectively to $\mathrm{x}$-y polarization for different concentration levels of adulteration from $0.5 \mu \mathrm{m}$ to $1.5 \mu \mathrm{m}$ wavelength. In fact, the confinement losses are $1200 \mathrm{~dB} / \mathrm{cm}, 1360 \mathrm{~dB} / \mathrm{cm}, 1450 \mathrm{~dB} / \mathrm{cm}, 1510 \mathrm{~dB} / \mathrm{cm}$ and $1600 \mathrm{~dB} / \mathrm{cm}$ respectively to $10-50 \%$ concentration level. From the figure, it is identified that $50 \%$ mixture level has a higher loss profile of $1600 \mathrm{~dB} / \mathrm{cm}$ and the $10 \%$ mixture level has a minimum loss performance of $1200 \mathrm{~dB} / \mathrm{cm}$ respectively to the following case that is described in Table. 2 and Table. 3. However, all the loss peaks are established at the perpendicular cross-section of their corresponding core and spp mode.

Fig. 9 delineates the amplitude sensitivity alteration as a function of wavelength respectively for different density levels of adulteration from $0.5 \mu \mathrm{m}$ to $1.5 \mu \mathrm{m}$ operating wavelength. However, 


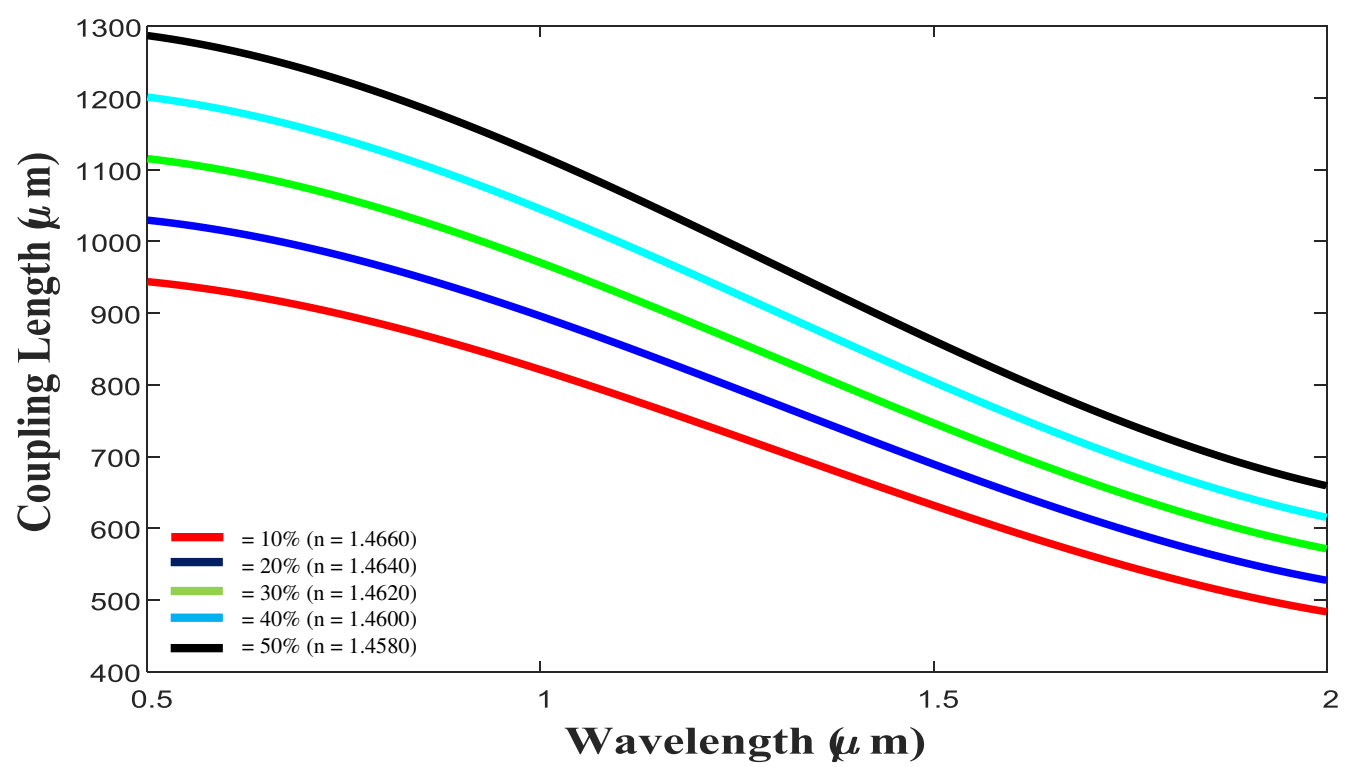

Fig. 5. The coupling length variation with respect to wavelength for oil adulteration sensing of the SPR-PCF structure for different concentrations of adulteration level.

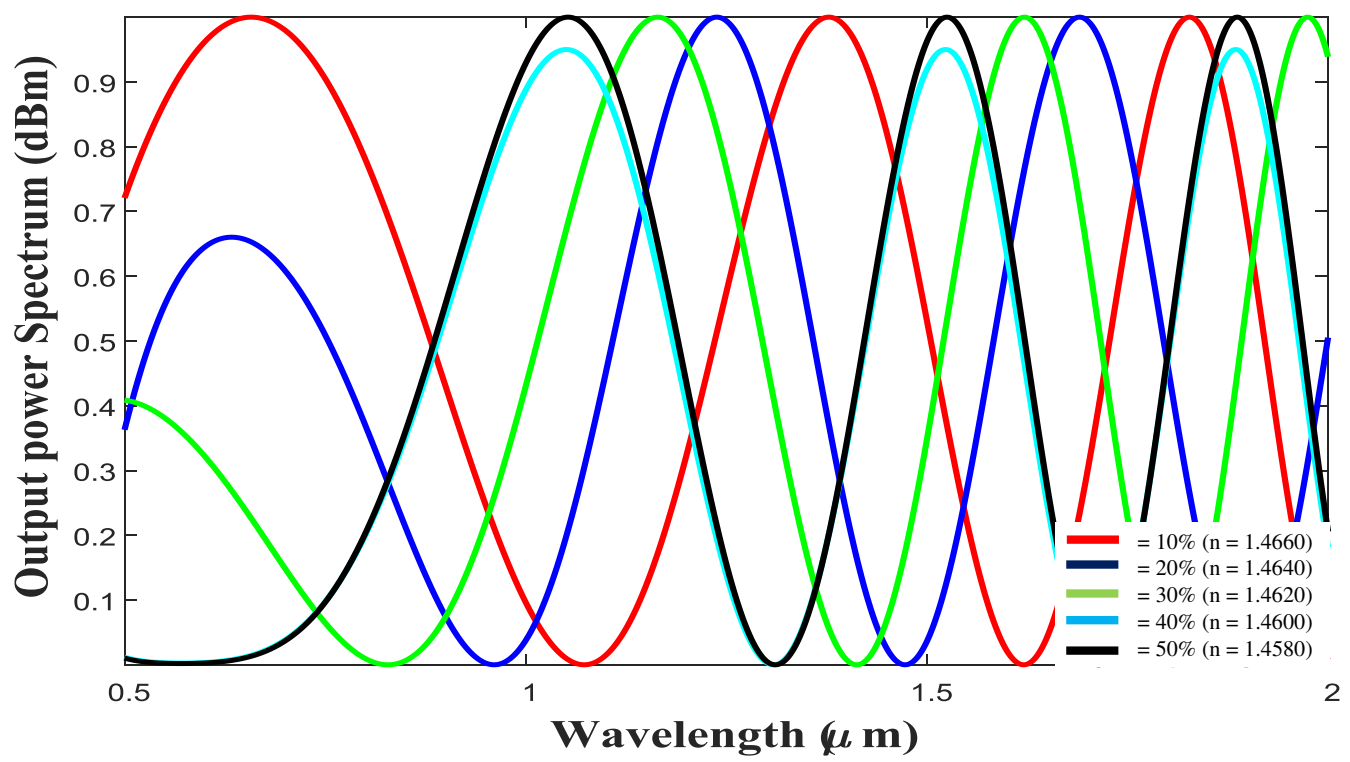

Fig. 6. The output power variation vs. wavelength for different concentrations of adulteration level of the proposed SPR-PCF.

from Table. 2 and Table. 3, the amplitude sensitivity of the SPR-PCF is $3550 R I U^{-1}, 4510$ $R I U^{-1}, 5902 R I U^{-1}, 7810 R I U^{-1}$ and $7980 R I U^{-1}$ respectively to $10 \%, 20 \%, 30 \%, 40 \%$ and $50 \%$. It can be noticed that $\mathrm{y}$-axis polarization has a greater amplitude profile compared to $\mathrm{x}$-axis polarization. It is noticed that the $50 \%$ adulteration level has a huge sensing performance of 7980 $R I U^{-1}$. On the other hand, the $10 \%$ adulteration level has a lesser sensing performance of 3550 $R I U^{-1}$ respectively for the following case.

Fig. 10 shows the maximum wavelength sensitivity and amplitude resolution vs. the variation 


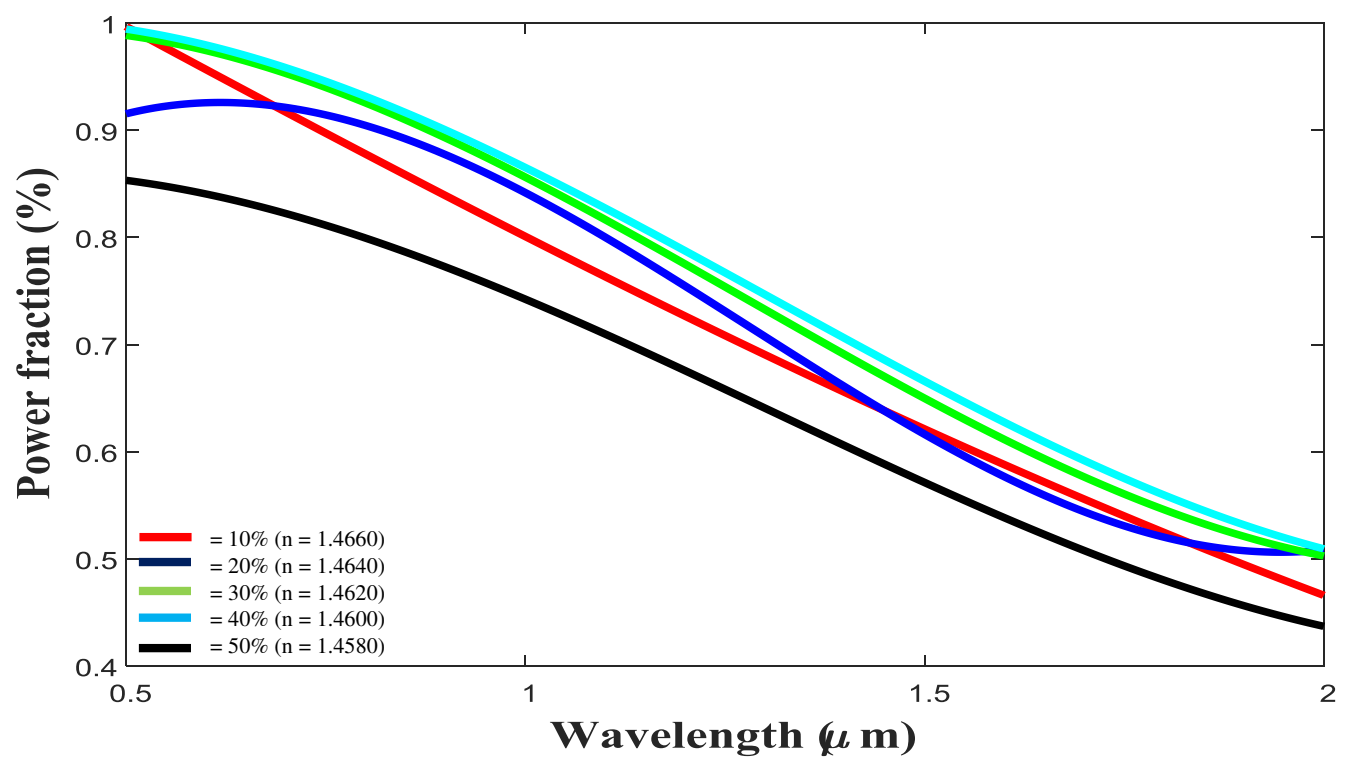

Fig. 7. The total power fraction with respect to wavelength for different concentrations of adulteration level of the proposed SPR-PCF.

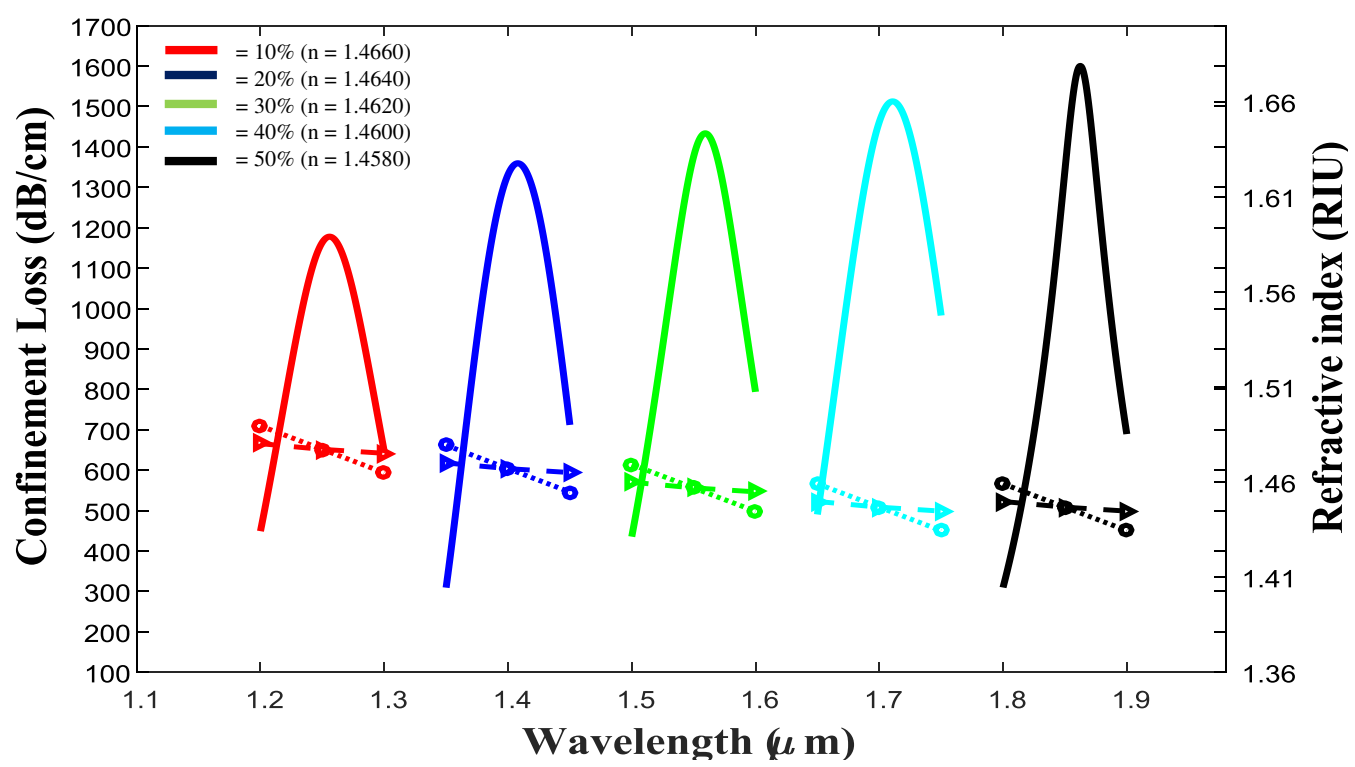

Fig. 8. The confinement loss profile vs. wavelength for different concentrations of adulteration level of the proposed structure.

of the refractive index. Conversely, the maximum possible wavelength sensitivity varies from $29611.76 \mathrm{~nm} / \mathrm{RIU}$ to $49941 \mathrm{~nm} / \mathrm{RIU}$ along with the resolution level that varies between $2.31 \times 10^{-4}$ RIU and $6.32 \times 10^{-4}$ RIU with respect to the RI variation of from 1.4580 to 1.4660 . It is noticed that the total resolution profile of the proposed sensor displays a smoother convex upward curve with respect to the refractive index. Alternatively, wavelength sensitivity may vary with an upward concave trend for the RI variation. To sum up, the lower refractive index (RI) possesses to have lesser wavelength-sensitivity and the resolution for the proposed PCF described in Table. 2 and 


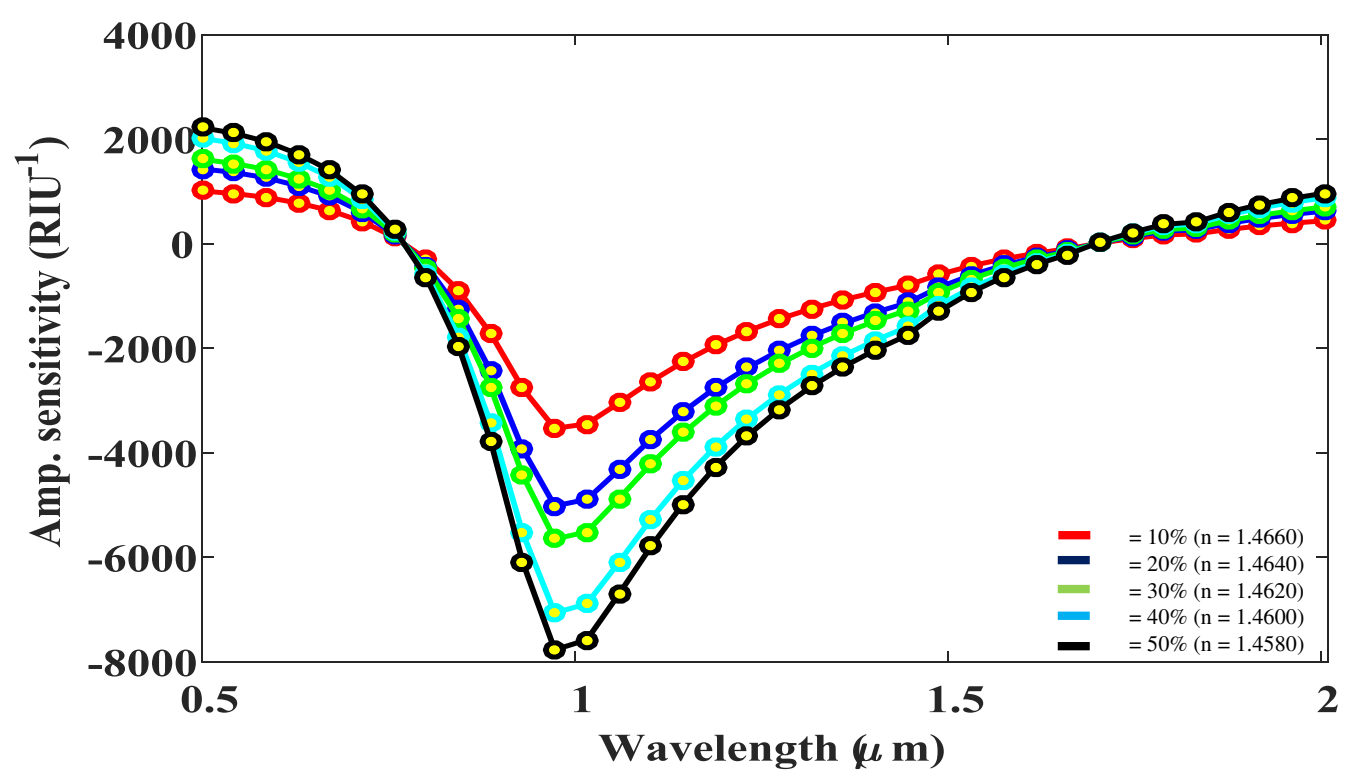

Fig. 9. The amplitude sensitivity comparison to the operating wavelength for different concentrations of adulteration level of the proposed PCF for x polarization.

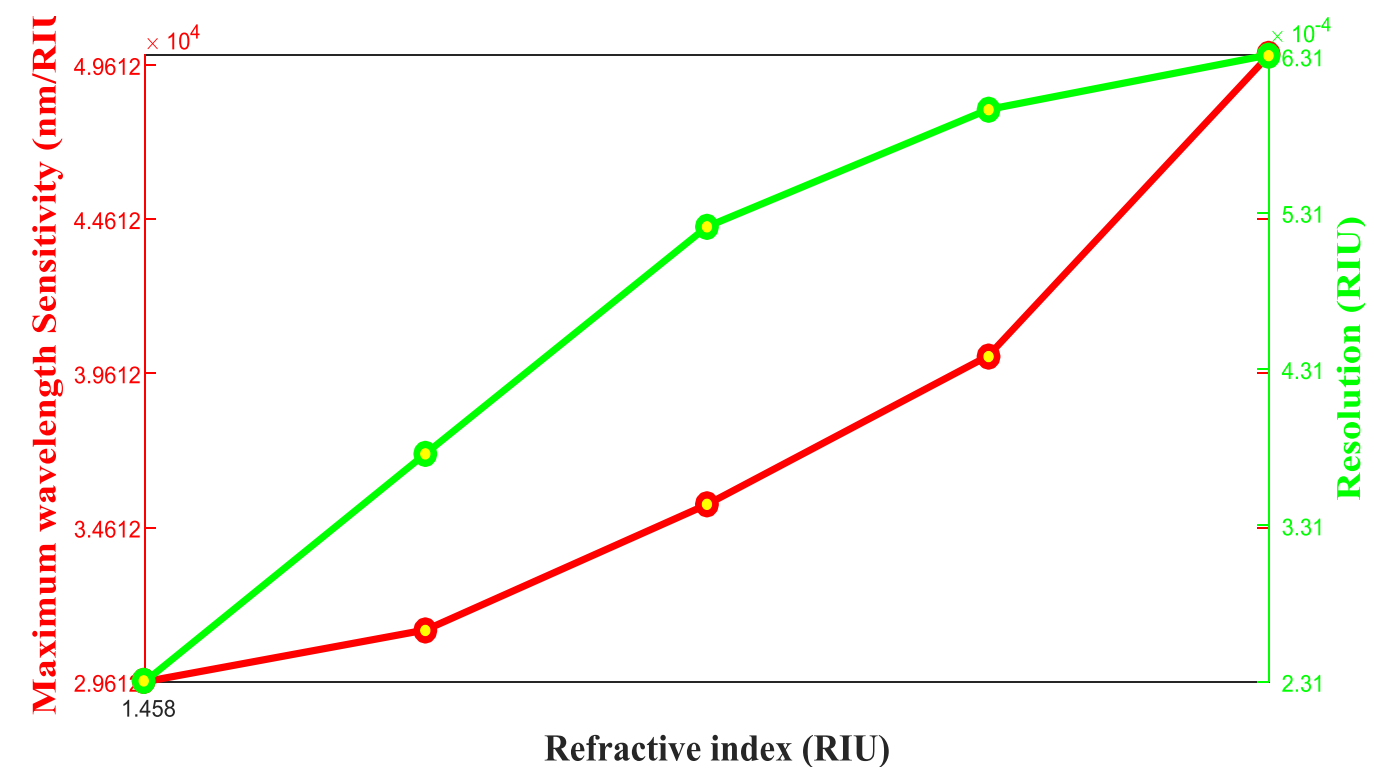

Fig. 10. The wavelength-sensitivity and resolution compared to the effective RI for different concentrations of adulteration level of the proposed SPR-PCF.

Table. 3 respectively.

Fig. 11 delineates the transmittance profile as a function of optical wavelength for the proposed sensor. Although, the transmittance profile may alter from $0 d B$ to $-180 d B$ having a steep declining peak for each concentration level. Therefore, the optimum possible transmittance performances are $-180 d B,-140 d B,-120 d B,-115 d B$ and $-105 \mathrm{~dB}$ respectively to $10-50 \%$ adulteration level. It is commented that the maximal transmittance profile is $-180 \mathrm{~dB}$ at the $10 \%$ mixture level that promotes more sensitivity and the lower transmittance is $-105 d B$ at the $50 \%$ mixture level 


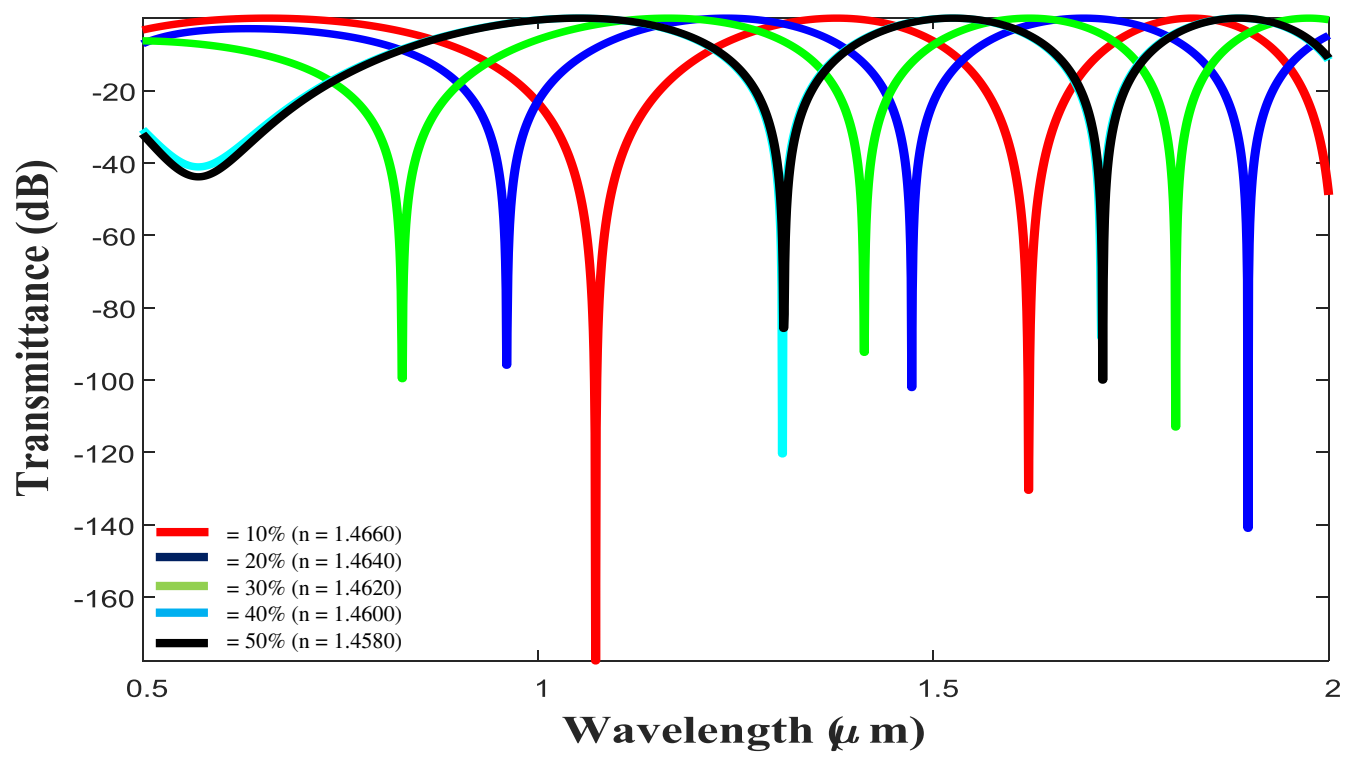

Fig. 11. The transmittance performance vs. the operating wavelength of the proposed PCF structure for different concentration levels of adulteration.

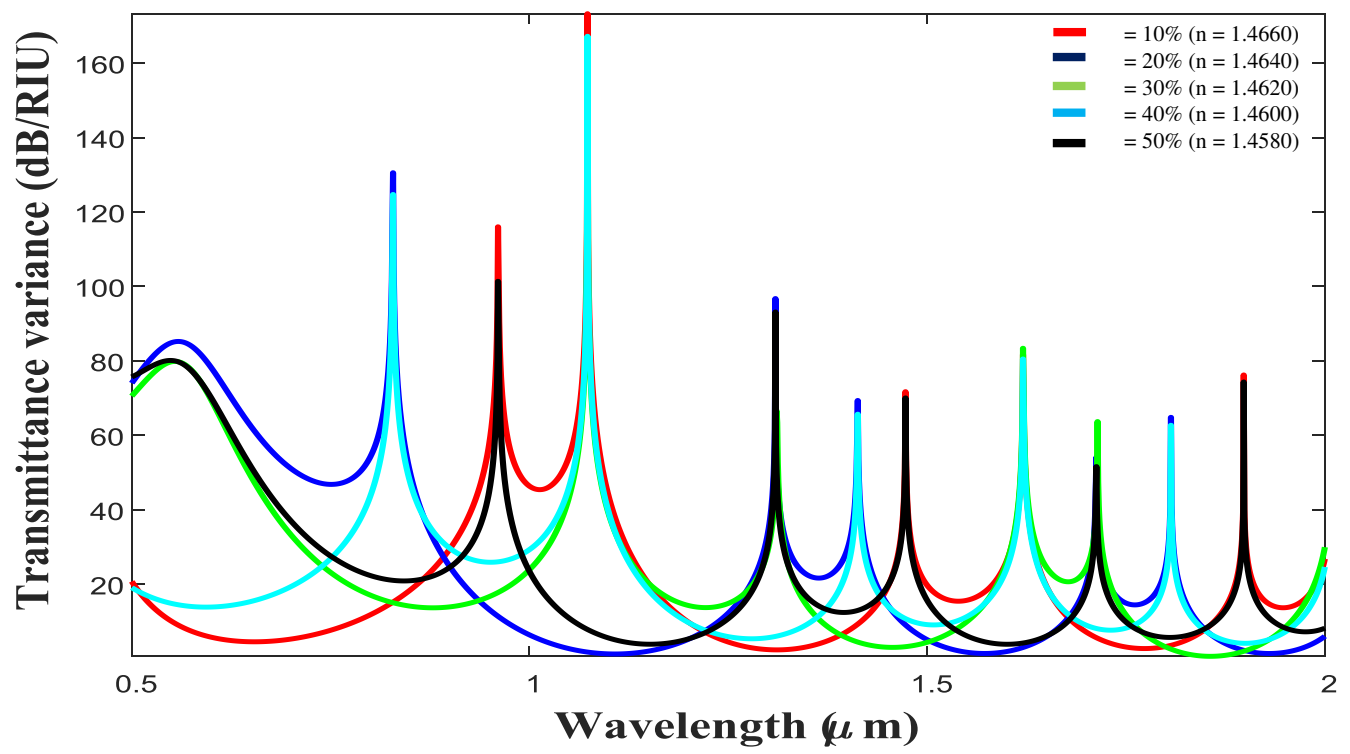

Fig. 12. The transmittance variance performance respectively to the wavelength of the proposed structure for different concentration levels of adulteration.

that promotes less sensitivity and so on.

However, transmittance-variance vs wavelength is displayed in Fig. 12. And, the entire transmittancevariance might range between $110 \mathrm{~dB} / R I U$ and $170 \mathrm{~dB} / R I U$ having a steep inclining peak for each estimate. Moreover, transmittance-variance of the suggested sensor is $170 \mathrm{~dB} / \mathrm{RIU}, 135$ $\mathrm{dB} / \mathrm{RIU}, 165 \mathrm{~dB} / R I U, 165 \mathrm{~dB} / R I U$ and $110 \mathrm{~dB} / R I U$ respectively to $10 \%, 20 \%, 30 \%, 40 \%$ and $50 \%$ adulteration level. It is noticed that $10 \%$ has higher transmittance-variance of $170 \mathrm{~dB} / \mathrm{RIU}$ and $50 \%$ has a lower transmittance-variance of $110 \mathrm{~dB} / \mathrm{RIU}$. In fact, a higher percentage of 
adulteration has a better average profile than others in terms of transmittance variance.

Fig. 13 shows the relative sensitivity responses for alteration comparison with respect to operating wavelength for the PCF based SPR. It depicts that the maximum possible sensitivity of $97.25 \%, 97.19 \%, 97.15 \%, 97.08 \%$, and $97.06 \%$ for respectively adulteration level from $10 \%$ to $50 \%$. Therefore, it depicts an upward shifting of sensing and continues up to the end in a convex manner. As a result, the minimum adulteration level provides a more relative sensing profile than the maximum adulteration levels and so on.

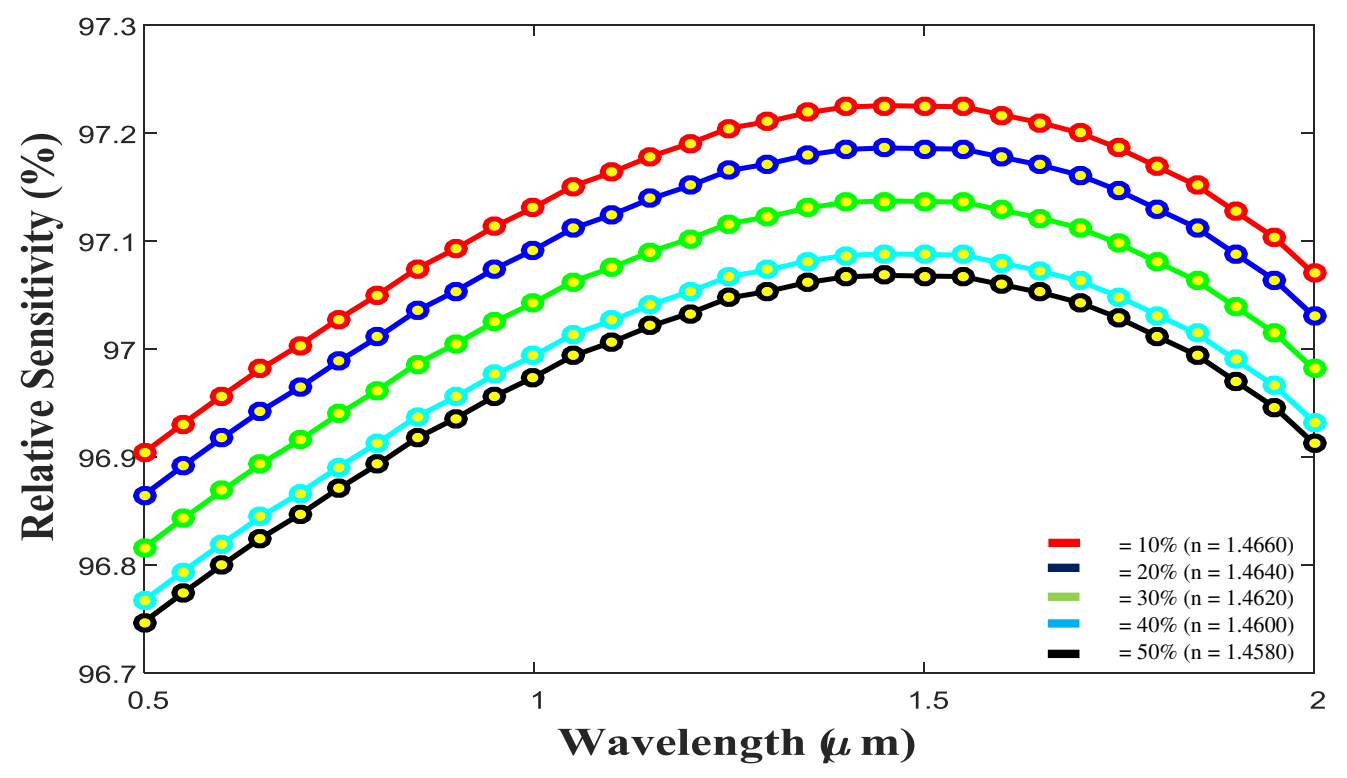

Fig. 13. The relative-sensitivity profile vs. operating wavelength for different mixture level of the proposed PCF.

Fig. 14 defines as the Figure-Of-Merit (FOM) vs. operating wavelength. Moreover, the most optimum FOM performances are 940, 945, 950, 970 and 980 respectively to the maximum detection limit of 0.0020. So, it is noticed that higher adulteration level of fuels has more FOM than others from Table. 3. Here, the greater value of FOM confirms the better efficiency of the introduced sensor.

Fig. 15 defines the regression model of adulteration vs. the variation of RI. Furthermore, the more fitting of a regression sample indicates the more better performance indication for a higher linearly response. And, the resonance of fitting line varies according to the real part of RI as optimum parameters. So, the fitting functions are described as- $y=7.05 x+0.0034, y=$ $7.06 x+0.0034, y=7.04 x+0.0034, y=7.07 x+0.0034$ and $y=7.03 x+0.0034$ with $R^{2}$ values of $0.9831,0.9842,0.9719,0.9852$ and 0.9702 respectively to the $10 \%$ to $50 \%$ adulteration level as an optimum parameter. So, the lower adulteration level has a better fitting function than the higher adulteration level. The better fitting function in low precision adulteration level provides evidence of more accuracy of a SPR sensor. As a result, this sensor can be applied in the areas of aqueous analyte sensing or bio-sensing purposes where a low precision is so important. 


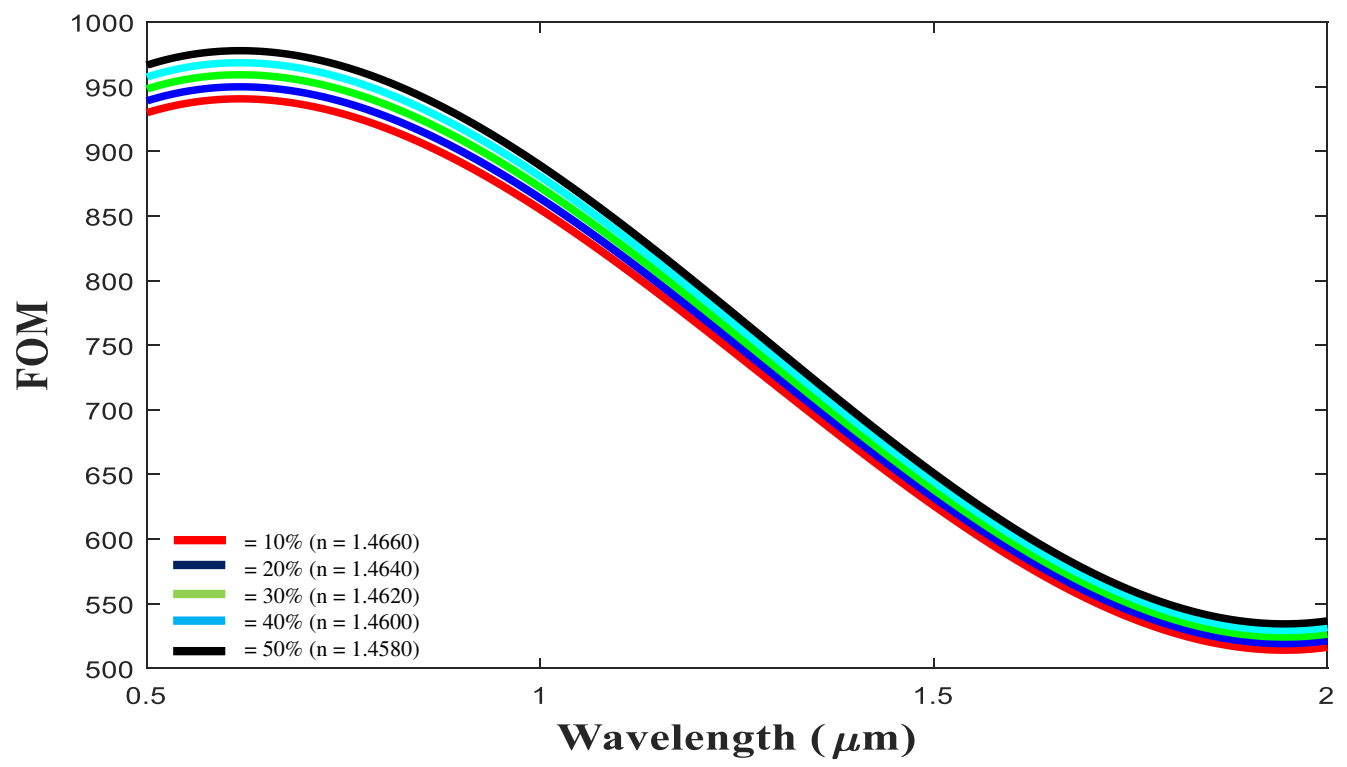

Fig. 14. The FOM vs. operating wavelength for the proposed PCF sensor for different mixture level.

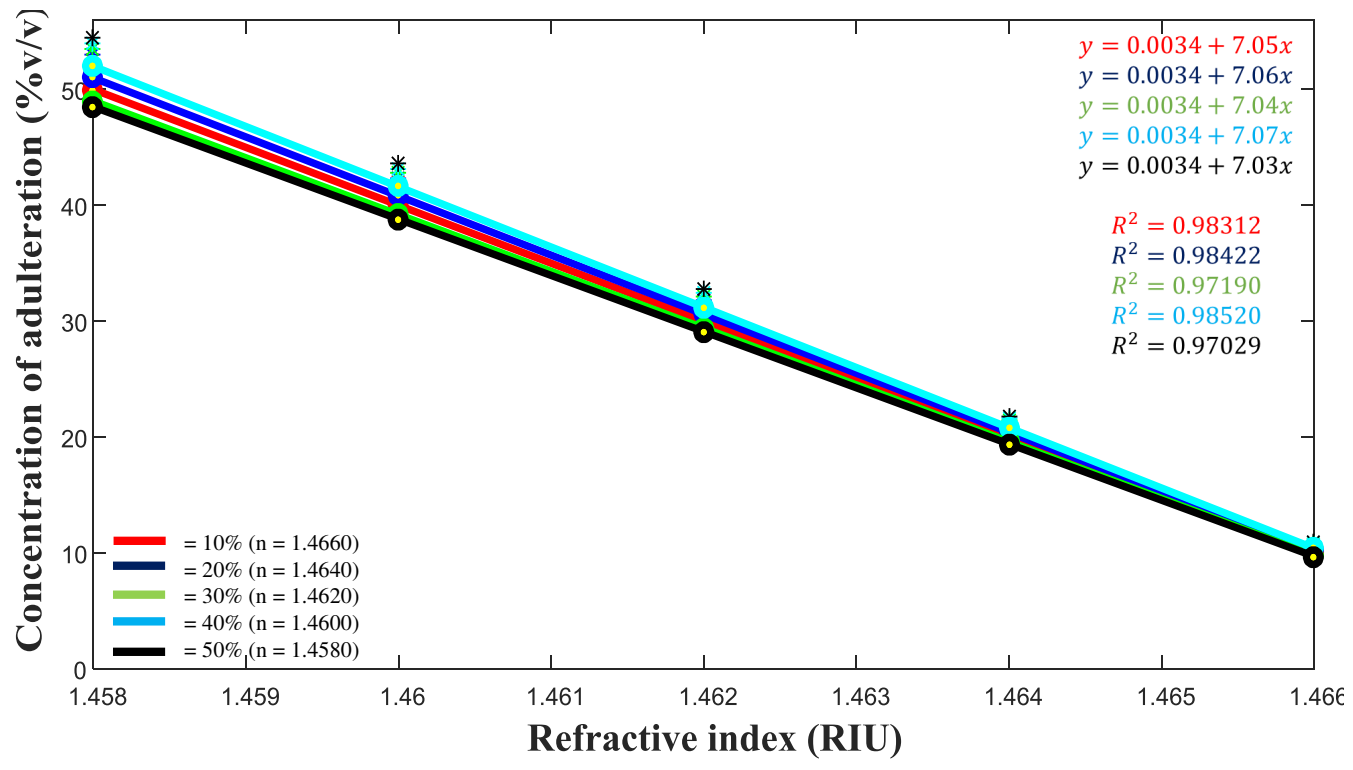

Fig. 15. The regression model of the concentration vs. refractive index for the proposed sensor for different mixture level.

\section{Conclusion}

In this letter, we propose a surface plasmon resonance (SPR) based biosensor for the detection of diesel adulteration for the first time in the field of bio-sensing. Though fabrication of the proposed model remains undone, the numerical analysis is done by COMSOL Multiphysics V-5.1 and fully simulation software MATLAB-V16 on 30190 mesh elements, 2792 boundary elements, and 206593 degrees of freedom using finite element method (FEM) at $25^{\circ} \mathrm{C}(298 \mathrm{~K})$ for different concentration level. However, the fabrication of the PCF can be done by using existing technologies 
like- sol-gel technique etc. Moreover, the entire experiment on SPR-PCF is justified through more than 12 the major optical measurements with tremendous results $-2.5 \times 10^{-3}, 1300 \mu \mathrm{m}, 99.93 \%$, $1200-1600 \mathrm{~dB} / \mathrm{cm},-7950 \mathrm{RIU} \mathrm{U}^{-1}, 49941.17 \mathrm{~nm} / \mathrm{RIU}, 6.32 \times 10^{-4} \mathrm{RIU},-180 \mathrm{~dB}, 170 \mathrm{~dB} / \mathrm{RIU}$, $97.25 \%, 980$, and 0.98312 respectively to the modal birefringence, coupling-length, modal powerfraction, confinement-loss, amplitude-sensitivity, wavelength-sensitivity, resolution, transmittance profile, transmittance-variance, relative-sensitivity, FOM and total resonance etc. In summary, the proposed sensor might be able to establish itself as a tough competitor in the area of optical sensing.

Acknowledgments The authors are grateful to all of the subjects who have contributed to this research. We thank the Science and Technology Unit at Umm Al-Qura University for their continued logistics support.

Authors' Contributions Conceptualization, K. Ahmed; data curation, formal analysis, investigation, methodology, M.A. Jabin, M.J. Rana; funding acquisition, F.A.A. Zahrani, F.M. Bui; project administration, K. Ahmed; resources, software, F.A.A. Zahrani; supervision, K. Ahmed; validation, B.K. Paul, K. Ahmed, F.M. Bui; visualization, M.A. Jabin, M.J. Rana, B.K. Paul, K. Ahmed; writing—original draft, M.A. Jabin, B.K. Paul, K. Ahmed, F.M. Bui; writing-review editing, M.A. Jabin, K Ahmed, F.A.A. Zahrani, F.M. Bui.

Funding The work is funded by grant number 12-INF2970-10 from the National Science, Technology and Innovation Plan (MAARIFAH), the King Abdul-Aziz City for Science and Technology (KACST), Kingdom of Saudi Arabia. This work was also supported in part by funding from the Natural Sciences and Engineering Research Council of Canada (NSERC).

Data Availability Data will be available upon request to the corresponding author.

\section{Compliance with Ethical Standards}

Conflict of interest There is no competing interest with other authors.

Ethical Approval Not applicable. (There is no human related data. So, ethical approval is not taken from the external body of the committee.)

Consent to Participate Not applicable. (There is no human related data. So, consent is not necessary to take from the participant.)

Consent to Publish Not applicable. (There is no human related data. So, consent to publish is not necessary to take from the participant.)

\section{References}

[1] M.R. Hasan et al.,"Spiral photonic crystal fiber-based dual-polarized surface plasmon resonance biosensor," IEEE SensorsJournal, vol. 18, pp. 133-140, 2018.

[2] D. J. J. Hu and H. P. Ho, "Recent advances in plasmonic photonic crystal fibers: design, fabrication and applications,” Adv. Opt. Photon., vol. 9, pp. 257-314, 2017. 
[3] M. A. Jabin et al, "Surface Plasmon Resonance Based Titanium Coated Biosensor for Cancer Cell Detection," IEEE Photonics Journal, vol. 1-1, 2019, http://dx.doi.org/10.1109/jphot.2019.2924825.

[4] M. A. Jabin et al., "Titanium-Coated Dual-Core D-Shaped SPR-Based PCF for Hemoglobin Sensing," Plasmonics, pp.1-10, 2019, http://dx.doi.org/10.1007/s11468-019-00961-6.

[5] X. Yang et al., "A photonic crystal fiber glucose sensor filled with silver nanowires," Optics Communications., vol. 359, pp. 279-284, 2016.

[6] E.K. Akowuah et al., "Numerical analysis of a photonic crystal fiber for biosensing applications," IEEE J. Quantum Electron. 48 (2012) 1403 -1410,2012, http://dx.doi.org/10.1109/JQE.2012

[7] R. Otupiri et al., "A novel birefrigent photonic crystal fibre surface plasmon resonance biosensor,” IEEEPhoton. J. 6 (2014), http://dx.doi.org/10.1109/JPHOT.2014.2335716.

[8] M.Y. Azab, M.F.O. Hameed, and S.S.A. Obayya, "Multi-functional optical sensor based on plasmonic photonic liquid crystal fibers," Opt. Quant. Electron. 49 (2) 49.2017

[9] C. Mouvet et al., "Determination of simazine in water samples by waveguide surface plasmon resonance," Anl. Chim. Acta 338 (1997) 109-117.1997

[10] M.S. Islam et al., "A novel approach of spectroscopic identification of chemical using photonic crystal fibre in terahertz regime," IEEE Sensors J. 18 (2) ,p- 575 -582,2018, http://dx.doi.org/10.1109/JSEN.2017.2775642.

[11] T. D. Bradley, "Optical properties of low loss $(70 \mathrm{~dB} / \mathrm{km})$ hypocycloid- core kagome hollow core photonic crystal fiber for Rb and Cs based optical applications," J. Lightw. Technol, vol. 31 , no. 16, pp. 2752-2755, 8.2013.

[12] L. Hajbaand and A. Guttman, "Circulatingtumor-celldetectionandcapture usingmicrofluidicdevices," TrACTrendsAnal.Chem,vol.59,pp.9-16, 2014.

[13] T. Li et al, "Detection of Breast Cancer cells specially and accurately by an electrochemical method" Biosensors Bioelectron, vol. 25, no. 12, pp. 2686-2689, 2010.

[14] L. FR et al., "Detection of circulating tumor cells in breast Cancer with a refined immune magnetic nanoparticle enriched assay and nested-RT-PCR," Nanomed., Nanotechnol., Biol. Med, vol. 9, no. 7, pp. 1106-1113, 102013.

[15] R. Ritchie, "Plasma losses by fast electrons in thin films," Phys. Rev. 106, p. 874, 1957.

[16] B. Liedberg, C. Nylander and I. Lunström, "Surface plasmon resonance for gas detection and biosensing," Sensors Actuators 4 ,p. 299-304, 1983.

[17] J.N. Dash and R. Jha, "SPR biosensor based on polymer PCF coated with conducting metal oxide," IEEE Photon. Technol. Lett. 26,p. 595-598, 2014

[18] K.M. McPeak et al. "Plasmonic films can easily be better: rules and recipes," ACS Photon. 2 (2015) 326-333,2015

[19] A.A. Rifat, G.A. Mahdiraji, Y.G. Shee, M.J. Shawon, F.M. Adikan, "A novel photonic crystal fiber biosensor using surface plasmon resonance," Proc. Eng. 140, p- 1-7. Jan 2016 
[20] B. Shuai et al., "A multi-core holey fiber based plasmonic sensor with large detection range and high linearity," Opt.Express, vol. 20, pp. 5974-5986, 2012.

[21] C. Liu et al., "Numerical analysis of a photonic crystal fiber based on a surface plasmon resonance sensor with an annular analyte channel," OpticsCommunications, vol. 382, pp. 162166, 2017.

[22] E.K. Akowuah et al., "Numerical analysis of a photonic crystal fiber for biosensing applications," IEEE J. Quantum Electron. 48, pp. 1403-1410, 2012.

[23] J.N. Dash, and R. Jha, "SPR biosensor based on polymer PCF coated with conducting metal oxide," IEEE Photon. Technol. Lett. vol. 26, pp. 595-598, 2014.

[24] A. A. Rifat et al., "Photonic crystal fiber-based plasmonic biosensor with external sensing approach," J. Nanophoton., vol. 12, no. 1, p. 012503, Jun. 2017.

[25] M. Suganthy et al., "Analysis of optical sensitivity of Analytes in aqua solutions," Optik, 178, pp. 970-978, 2018.

[26] K. Ahmed et al., "Tetra-core Surface Plasmon Resonance based Biosensor for Alcohol Sensing," Physica B: Condensed Matter, 570, pp. 48-52, 2019.

[27] S. Chakma,et al., "Gold coated photonic crystal fiber biosensor based on surface plasmon resonance," Sens. and Bio-sens.. Res. Vol- 18, pp-7-12, 2018.

[28] N. Ayyanar et al., "Highly efficient compact temperature sensor using liquid infiltrated asymmetric dual elliptical core photonic crystal fiber," OpticalMaterials, vol. 64, pp. 574-582, 2017.

[29] J. Sultana et al., "Terahertz detection of alcohol using a photonic crystal fiber sensor," vol. 57, no. 10, pp. 2426-2432, 2018.

[30] A. K. Mishra, S. K. Mishra, and B. D. Gupta, "SPR based fiber optic sensor for refractive index sensing with enhanced detection accuracy and Figure of merit in the visible region," Opt. Commun. 344, 86-91, 2015.

[31] V. Mishra, V. Tiwaril and P.N. Patel, "Nanoporous Silicon Microcavity Based Fuel Adulteration Sensor", Silicon Springer, vol. 8, pp. 409-415, 2016. DOI 10.1007/s12633-015-9311-x. 

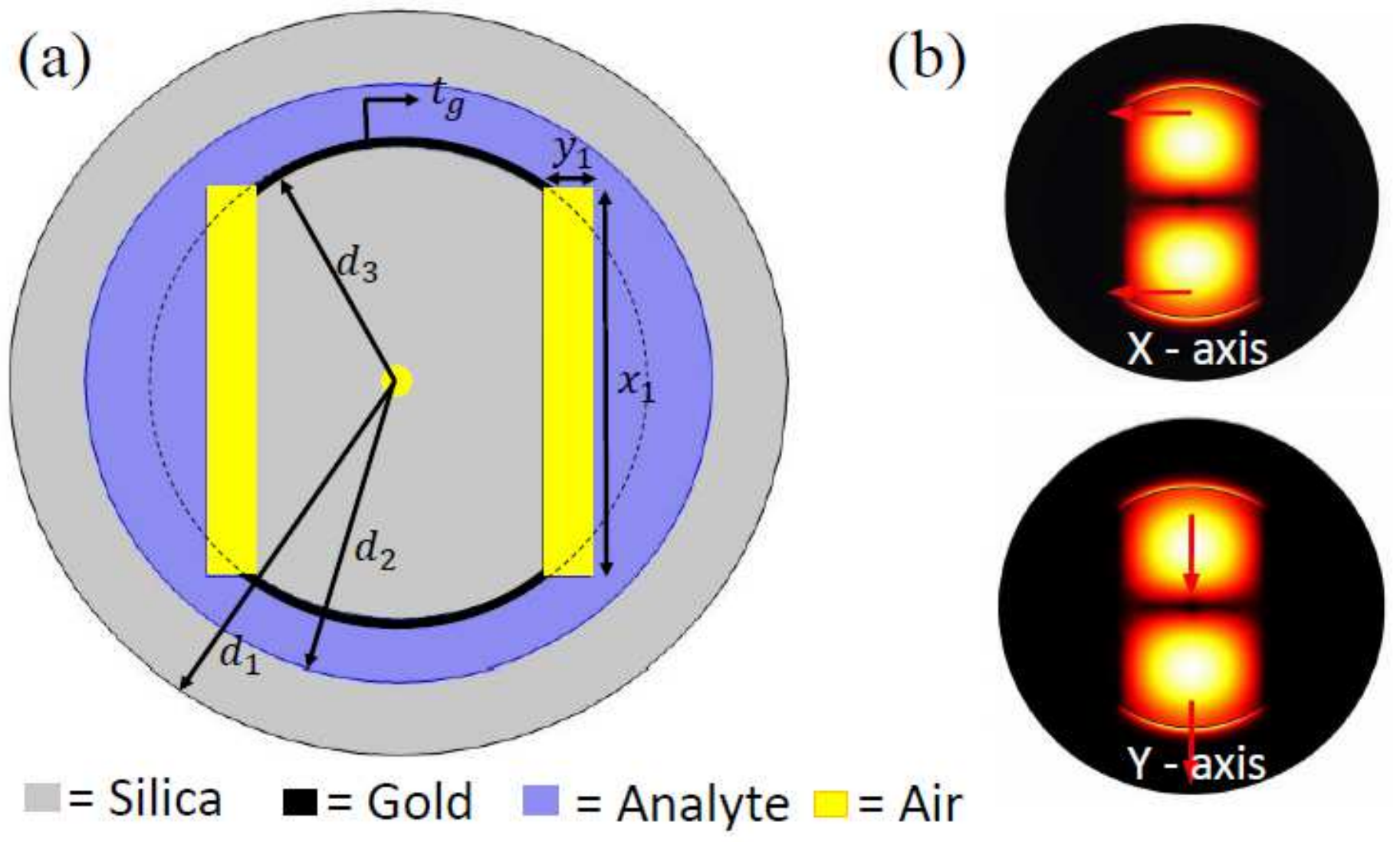

Figure 1

(a) The internal diagram of SPR-PCF diesel adulteration measurement bio-sensor along with its geometrical parameters likely as- $\mathrm{d} 1=5.80 \mu \mathrm{m}, \mathrm{d} 2=5.235 \mu \mathrm{m}, \mathrm{d} 3=4.60 \mu \mathrm{m}, \mathrm{tg}=35 \mathrm{~nm}, \mathrm{x} 1=3.80 \mu \mathrm{m}$, $y 1=0.50 \mu \mathrm{m}$ and pitch-constant $\mathrm{P}=2.0 \mu \mathrm{m}$. And, (b) The modes confinement of respectively for $\mathrm{x}$ and $\mathrm{y}-$ polarization. 


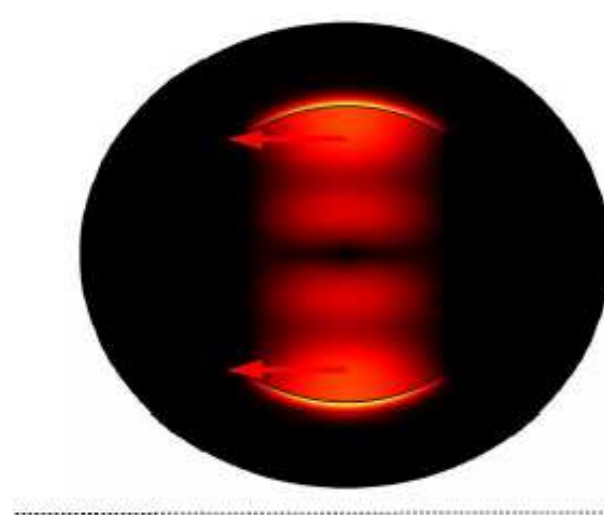

(a)

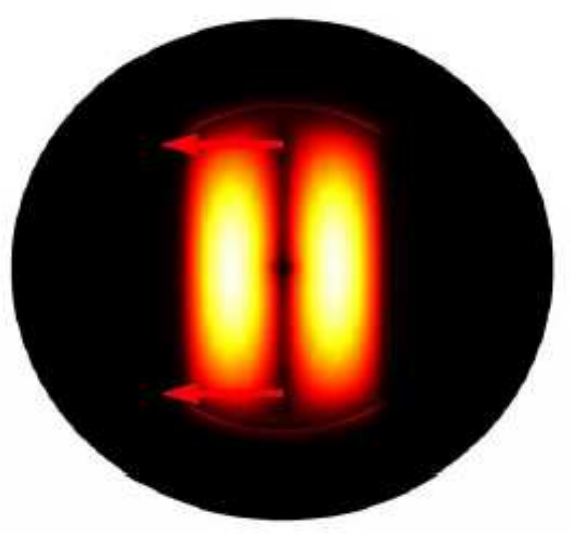

(c) (b)

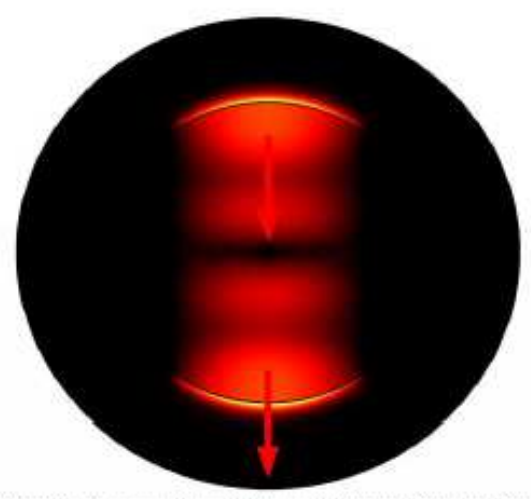

(d)

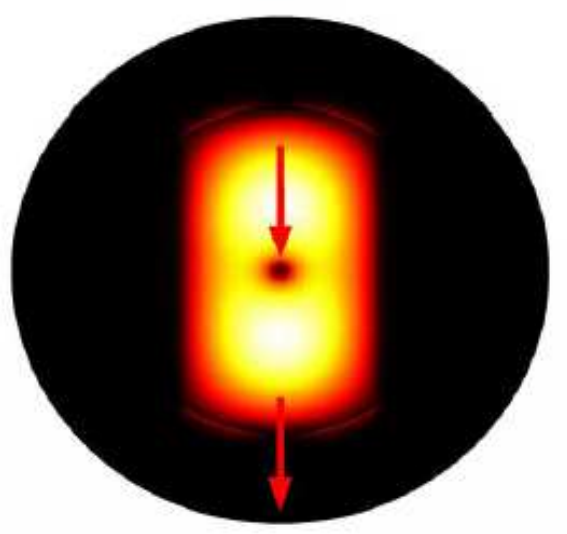

Figure 2

(a-b) The surface plasmon (SPP) mode and (c-d) The mode-confinement for the core of the proposed structures for $x-y$ axis polarization respectively. 


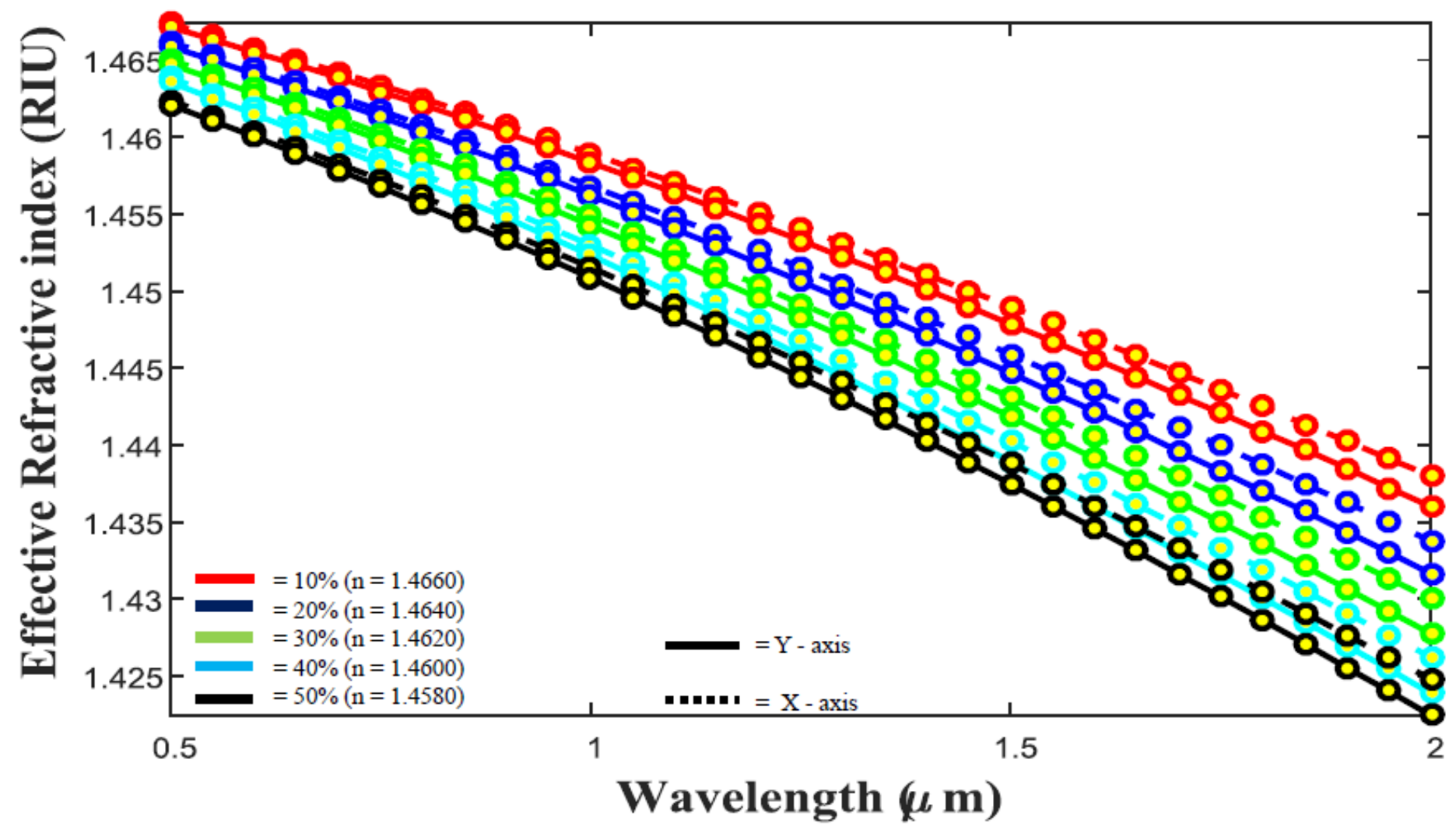

Figure 3

The effective refractive index (RI) profile vs. operating wavelength for the proposed sensor for $x-y$ axis polarization for different concentrations of adulteration level.

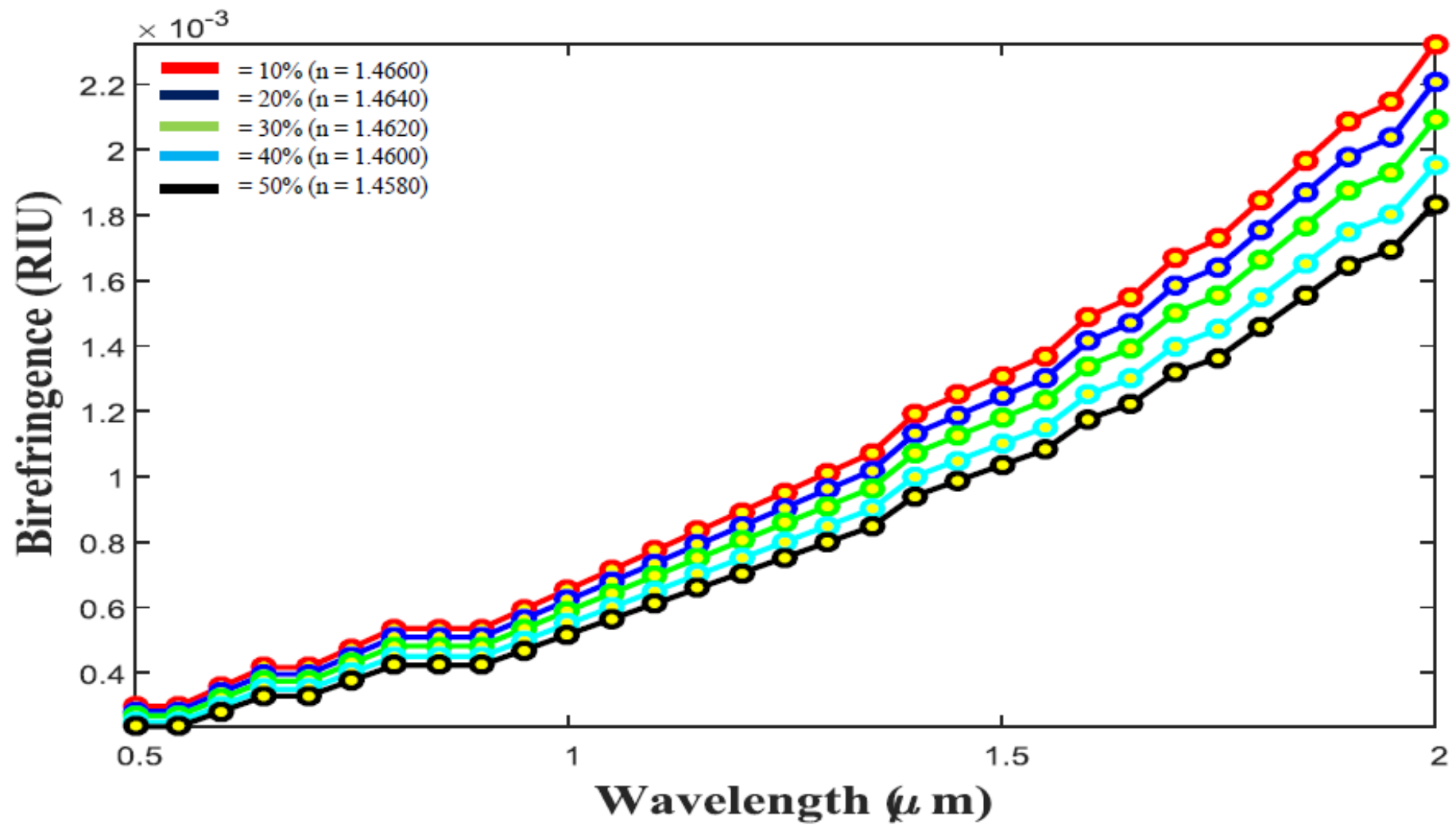




\section{Figure 4}

The modal-birefringence performance of the proposed SPR-PCF oil adulteration sensor for different concentrations of adulteration level.

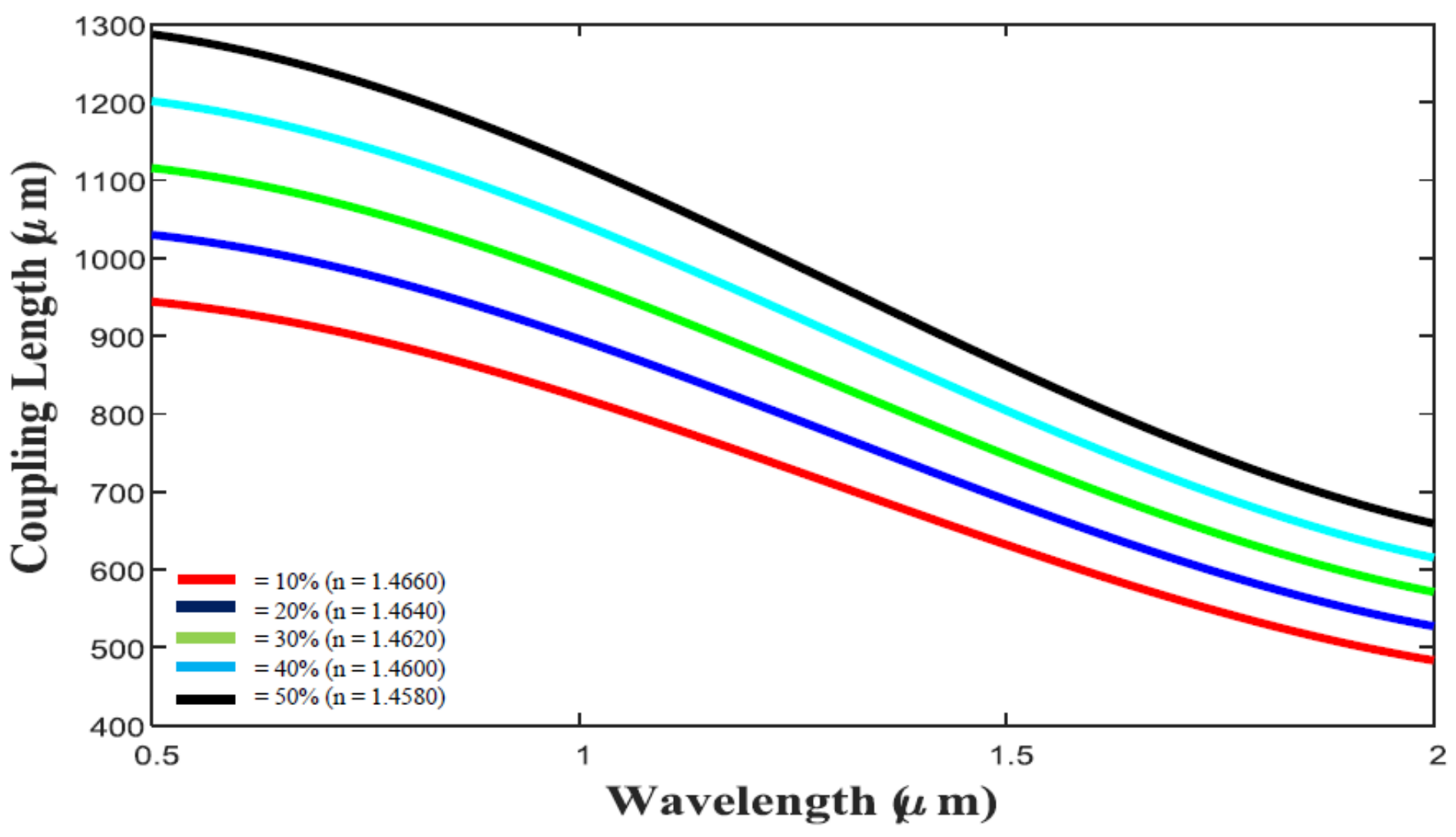

\section{Figure 5}

The coupling length variation with respect to wavelength for oil adulteration sensing of the SPR-PCF structure for different concentrations of adulteration level. 


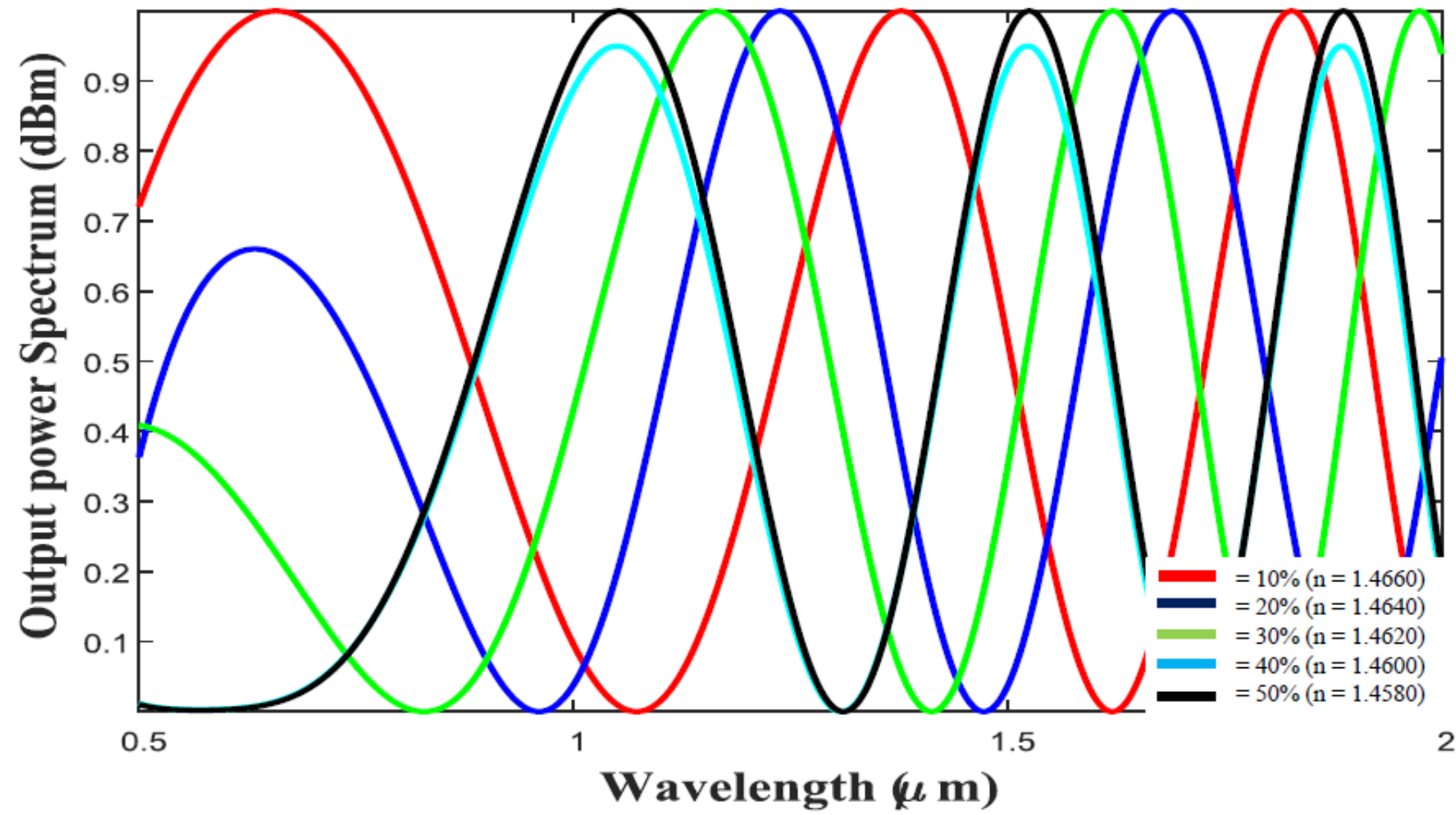

Figure 6

The output power variation vs. wavelength for different concentrations of adulteration level of the proposed SPR-PCF.

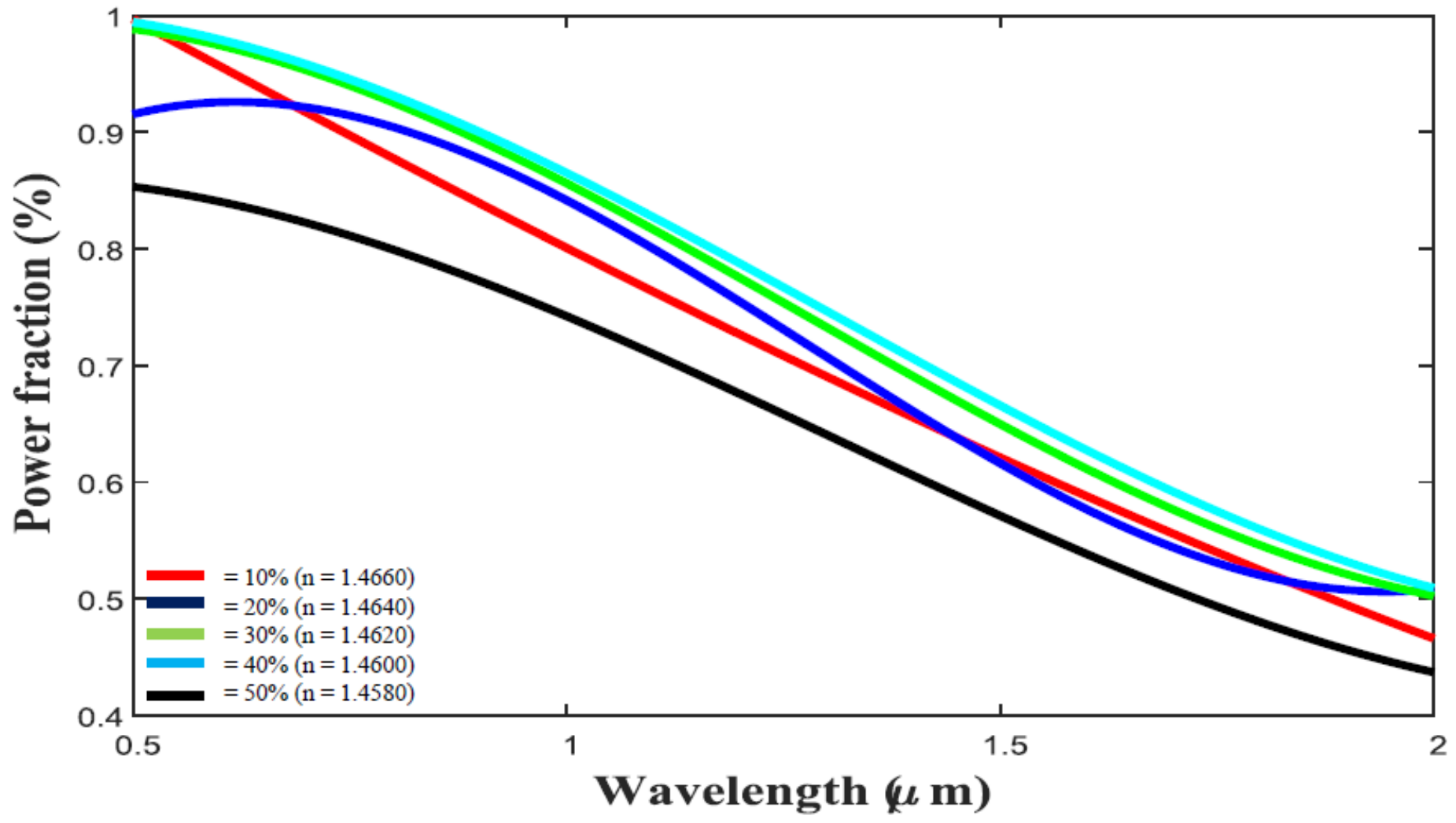


Figure 7

The total power fraction with respect to wavelength for different concentrations of adulteration level of the proposed SPR-PCF.

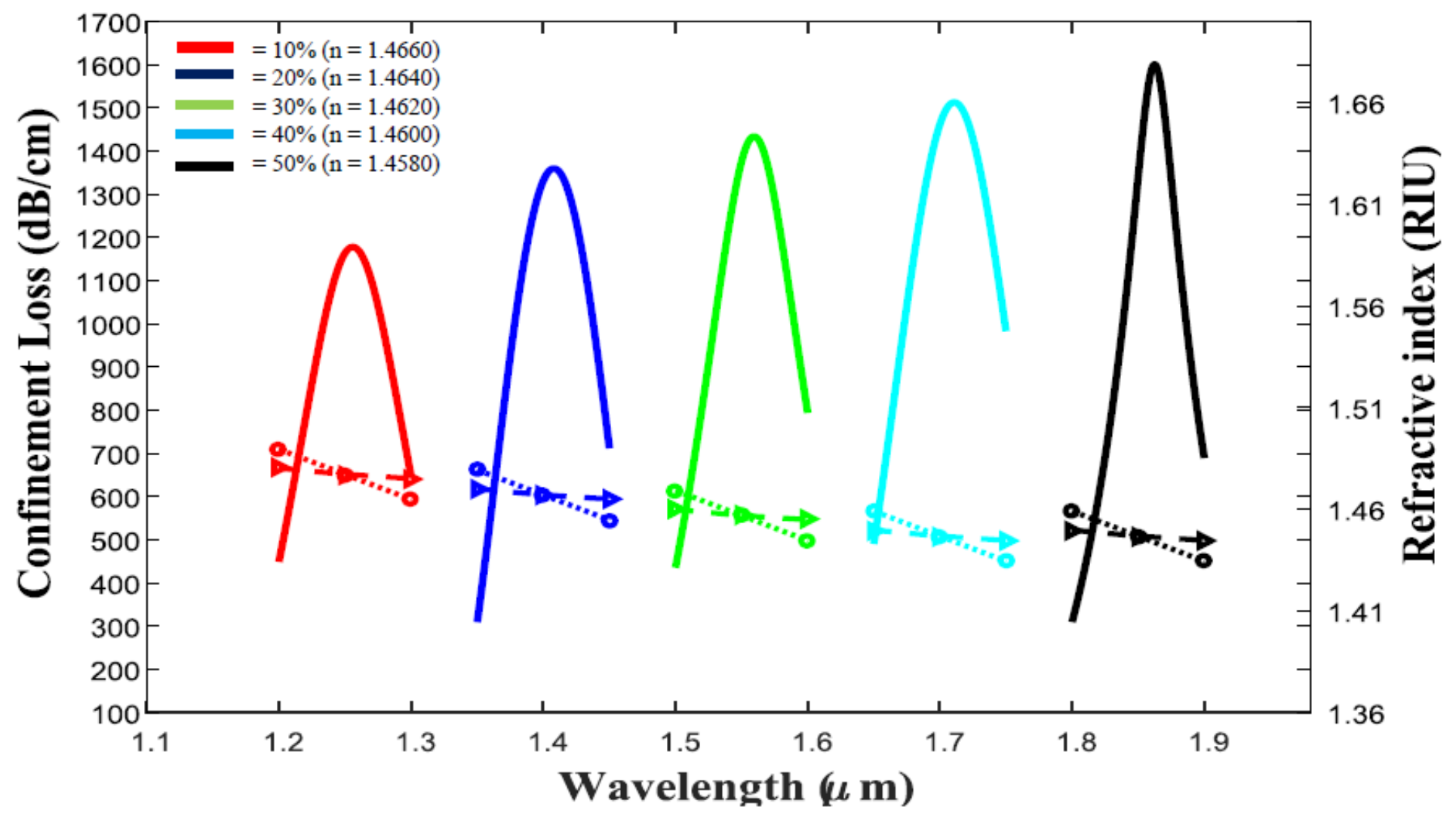

Figure 8

The confinement loss profile vs. wavelength for different concentrations of adulteration level of the proposed structure. 


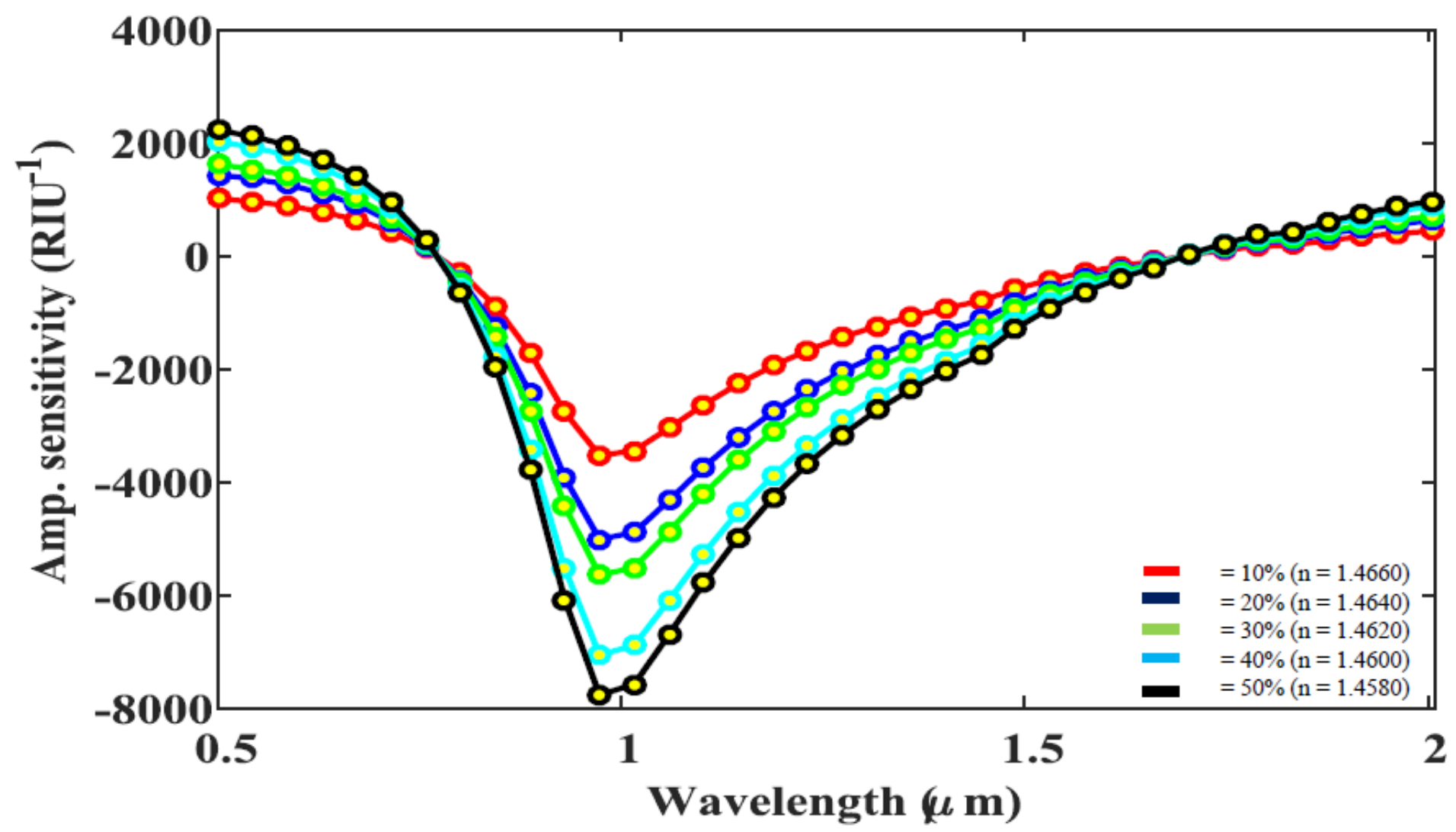

Figure 9

The amplitude sensitivity comparison to the operating wavelength for different concentrations of adulteration level of the proposed PCF for $\mathrm{x}$ polarization.

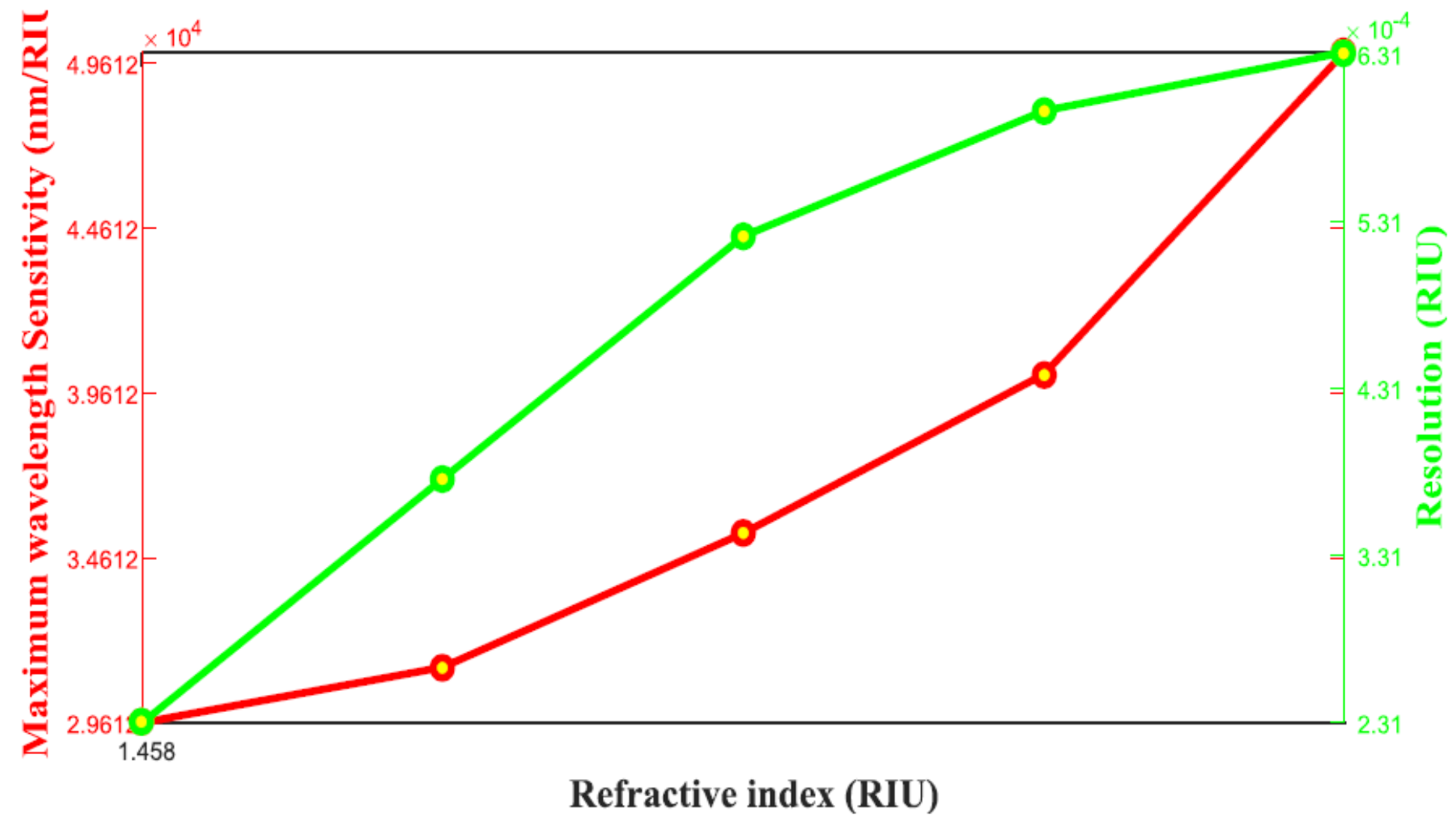


Figure 10

The wavelength-sensitivity and resolution compared to the effective RI for different concentrations of adulteration level of the proposed SPR-PCF.

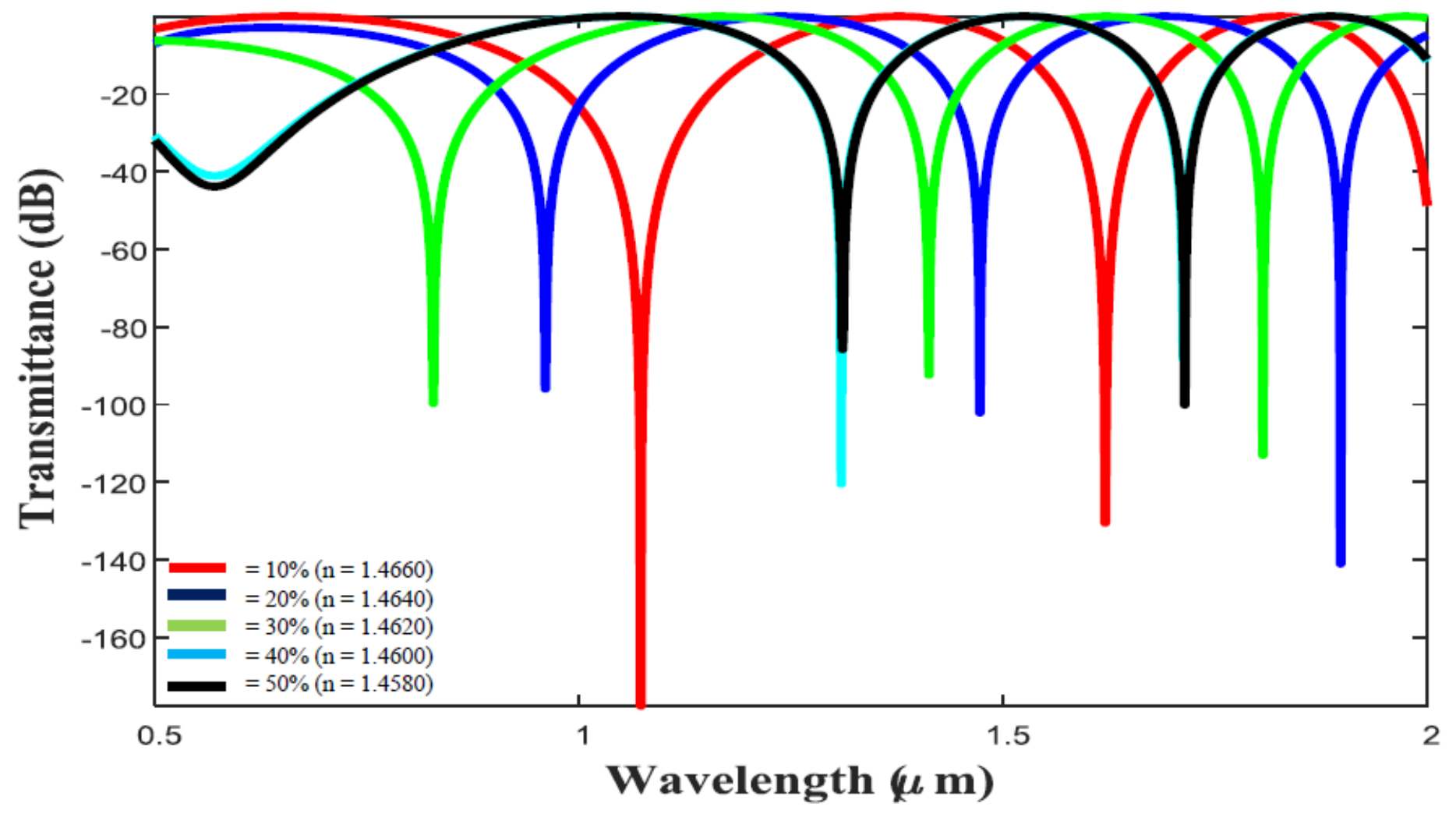

Figure 11

The transmittance performance vs. the operating wavelength of the proposed PCF structure for different concentration levels of adulteration. 


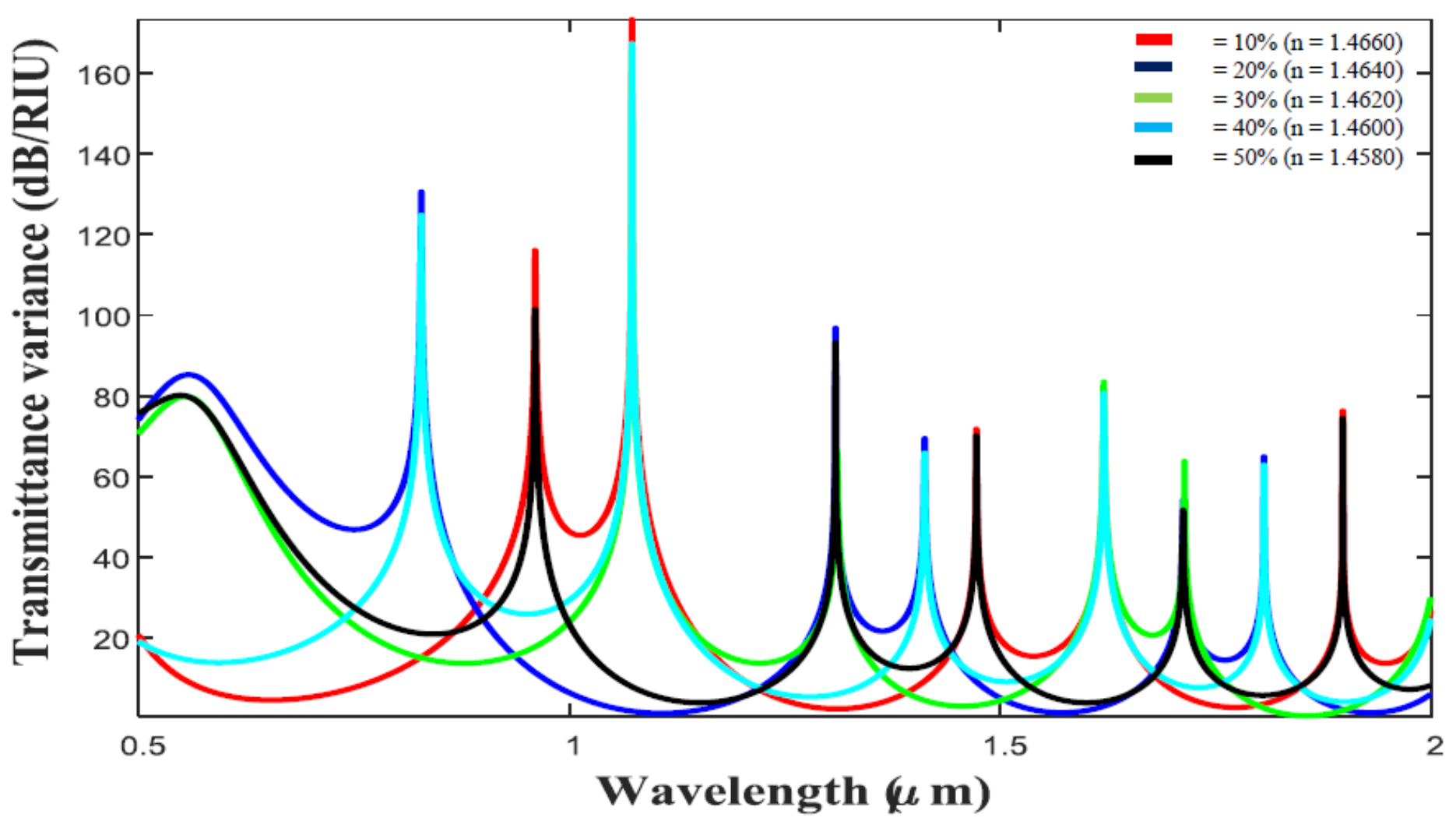

Figure 12

The transmittance variance performance respectively to the wavelength of the proposed structure for different concentration levels of adulteration.

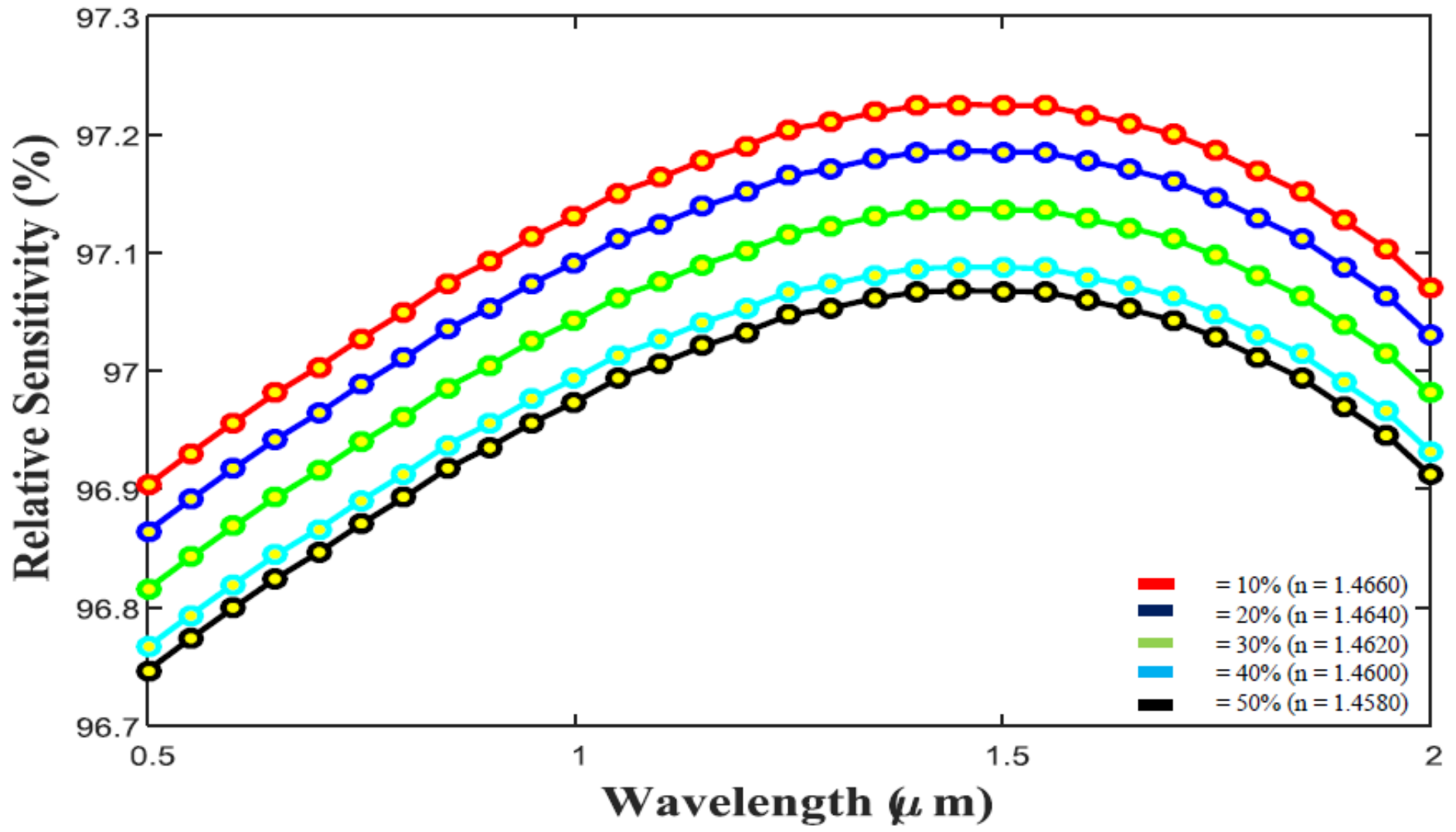


Figure 13

The relative-sensitivity profile vs. operating wavelength for different mixture level of the proposed PCF.

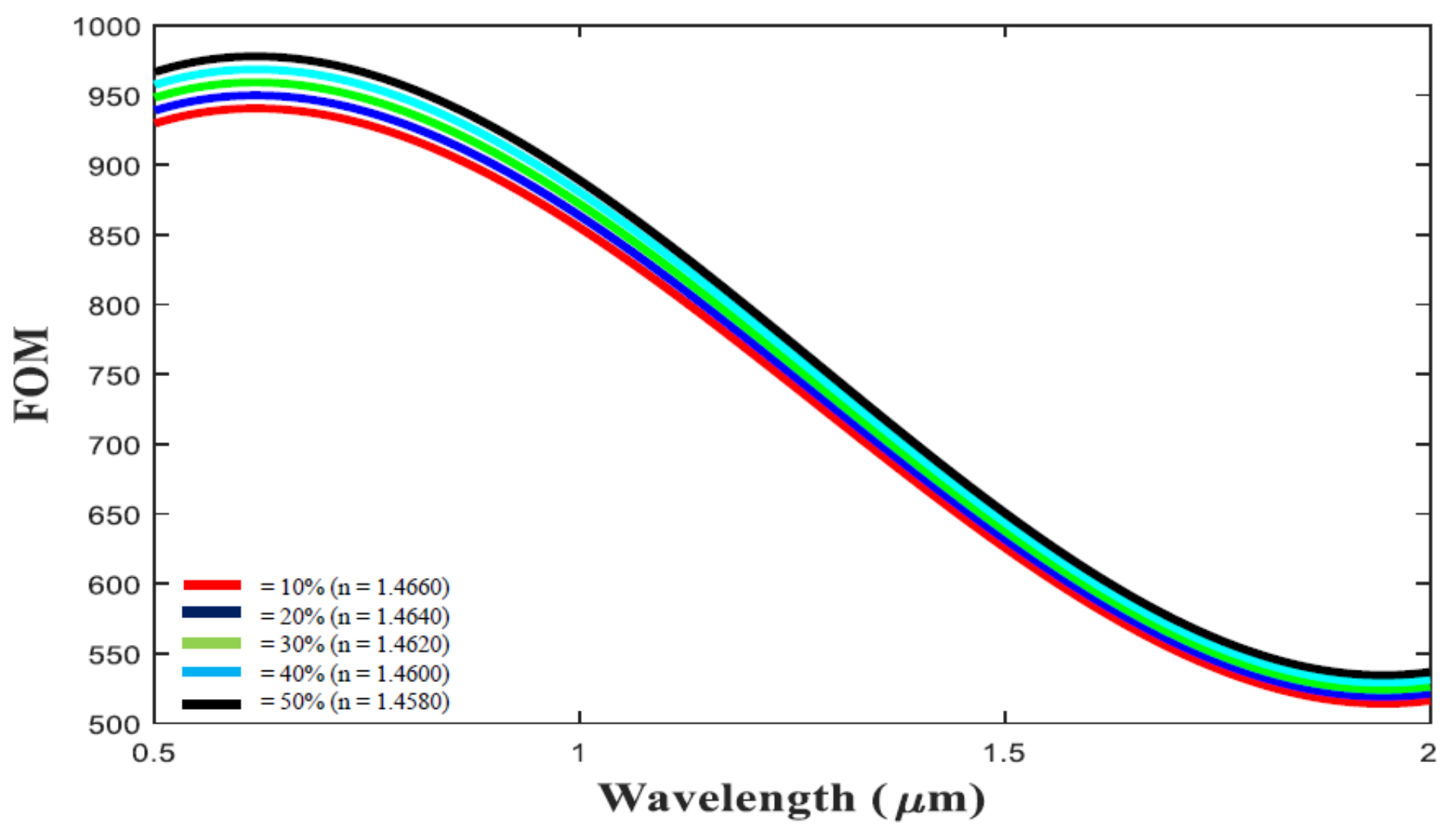

Figure 14

The FOM vs. operating wavelength for the proposed PCF sensor for different mixture level. 


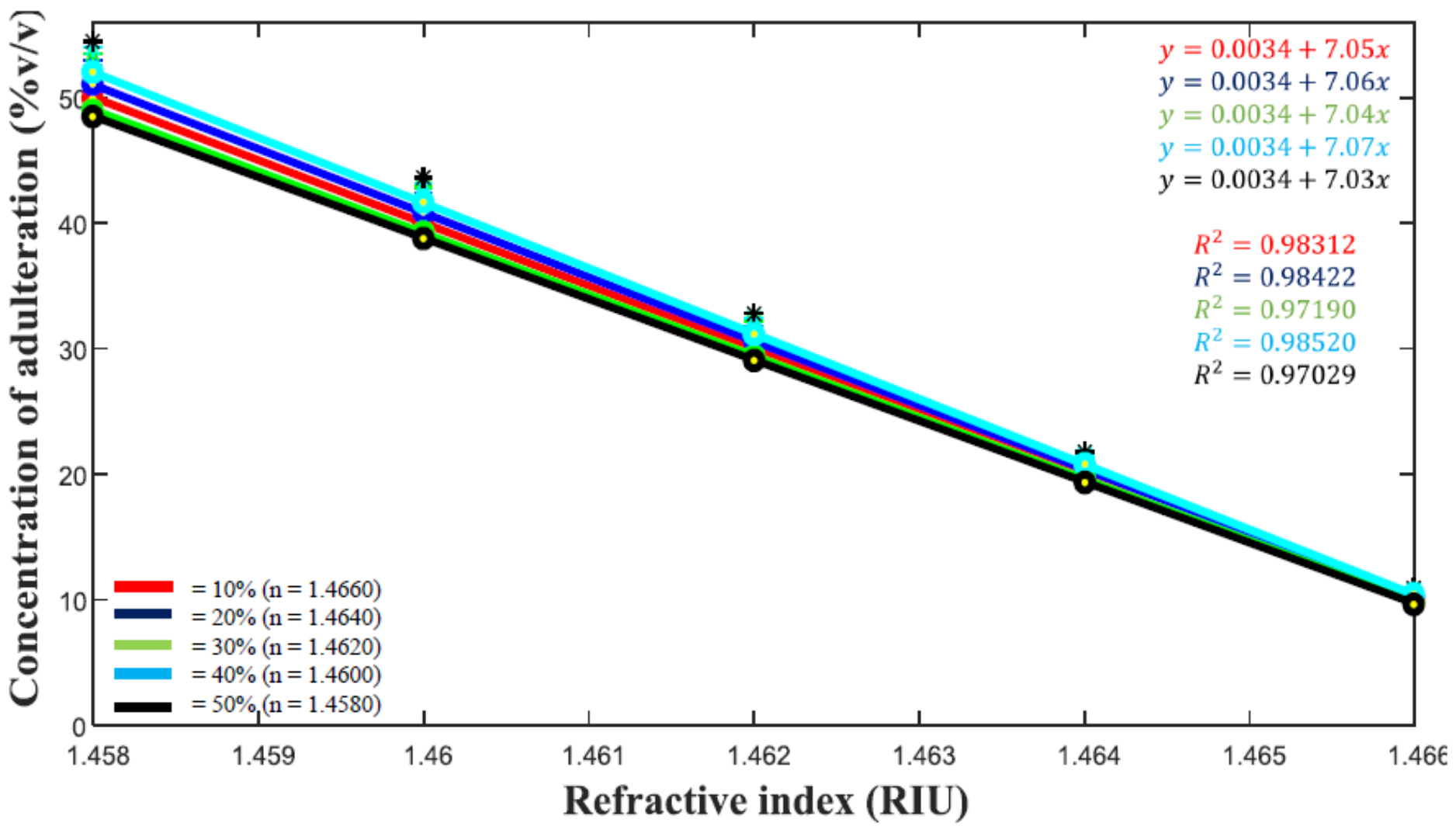

Figure 15

The regression model of the concentration vs. refractive index for the proposed sensor for different mixture level. 